Pacific

Journal of

Mathematics

\title{
THE GEOGRAPHY OF SYMPLECTIC 4-MANIFOLDS WITH DIVISIBLE CANONICAL CLASS
}

MARK J. D. HAMILTON 


\title{
THE GEOGRAPHY OF SYMPLECTIC 4-MANIFOLDS WITH DIVISIBLE CANONICAL CLASS
}

\author{
MARK J. D. HAMILTON
}

\begin{abstract}
We consider a version of the geography question for simply connected symplectic 4-manifolds that takes as an additional parameter the divisibility of the canonical class. We also find new examples of 4-manifolds admitting several symplectic structures that are inequivalent under deformation and self-diffeomorphisms of the manifold.
\end{abstract}

1. General restrictions on the divisibility of the canonical class 39

2. The generalized fibre sum 42

3. The knot surgery construction 44

4. Symplectic 4-manifolds with $c_{1}^{2}=0$

5. Generalized knot surgery 49

6. Spin symplectic 4-manifolds with $c_{1}^{2}>0$ and negative signature 52

7. Nonspin symplectic 4-manifolds with $c_{1}^{2}>0$ and negative signature $\quad 54$

8. Construction of inequivalent symplectic structures 56

9. Examples of inequivalent symplectic structures 65

10. Branched coverings $\quad 72$

11. Surfaces of general type and pluricanonical systems 75

12. Branched covering construction of algebraic surfaces with divisible canonical class $\quad 76$

$\begin{array}{lr}\text { Acknowledgment } & 81\end{array}$

$\begin{array}{ll}\text { References } & 81\end{array}$

We are interested in the geography of simply connected, closed, symplectic 4-manifolds whose canonical classes have a given divisibility. In general, the geography question aims at finding for any given pair of integers $(x, y)$ a closed 4-manifold $M$ with some a priori specified properties (for example, irreducible, spin, simply connected, symplectic or complex) such that the Euler characteristic $e(M)$ equals $x$ and the signature $\sigma(M)$ equals $y$. This question has been considered

MSC2000: 14J29, 57N13, 57R17.

Keywords: geography, symplectic, canonical class.

This work was supported by the Studienstiftung des deutschen Volkes and the Deutsche Forschungsgemeinschaft. 
for simply connected symplectic 4-manifolds both in the spin and nonspin case for example in [Gompf 1995; Park and Szabó 2000; Park 1998; 2002]; see also [Chen 1987; Fintushel and Stern 1994; Persson 1981; Persson et al. 1996]. We consider the geography question for simply connected symplectic 4-manifolds whose canonical class, considered as an element in second cohomology with integer coefficients, is divisible by a given integer $d>1$. Since the canonical class is characteristic, the first case $d=2$ corresponds to the general case of spin symplectic 4-manifolds.

Geography questions are often formulated in terms of the invariants $c_{1}^{2}$ and $\chi_{h}$ instead of $e$ and $\sigma$, which for smooth closed 4-manifolds are defined by

$$
c_{1}^{2}(M)=2 e(M)+3 \sigma(M) \quad \text { and } \quad \chi_{h}(M)=\frac{1}{4}(e(M)+\sigma(M)) .
$$

For complex 4-manifolds these numbers have the same value as the square of the first Chern class and the holomorphic Euler characteristic, making the definitions consistent.

The constructions we use here depend on generalized fibre sums of symplectic manifolds, which are also known as Gompf sums or normal connected sums [Gompf 1995; McCarthy and Wolfson 1994], in particular in the form of knot surgery [Fintushel and Stern 1998] and a generalized version of knot surgery along embedded surfaces of higher genus [Fintushel and Stern 2004]. Some details on the generalized fibre sum can be found in Section 2.

In Sections 4, 6 and 7, we consider the case $c_{1}^{2}=0$ and the spin and nonspin cases for $c_{1}^{2}>0$ and negative signature, while the case $c_{1}^{2}<0$ is covered at the end of Section 1. We do not consider the case of nonnegative signature, since even without a restriction on the divisibility of the canonical class, such simply connected symplectic 4-manifolds are known to be difficult to find.

As a consequence of these geography results, there often exist at the same lattice point in the $\left(\chi_{h}, c_{1}^{2}\right)$-plane several simply connected symplectic 4-manifolds whose canonical classes have pairwise different divisibilities. It is natural to ask whether the same smooth 4-manifold can admit several symplectic structures with canonical classes of different divisibilities; we consider this question in Sections 8 and 9. The symplectic structures with this property are inequivalent under deformations and orientation-preserving self-diffeomorphisms of the manifold. Similar examples have been found before on homotopy elliptic surfaces by McMullen and Taubes [1999], Smith [2000] and Vidussi [2001]. Another application of the geography question to the existence of inequivalent contact structures on certain 5-manifolds can be found in [Hamilton 2008].

In the final part of this article, we give an independent construction of simply connected symplectic 4-manifolds with divisible canonical class by finding complex surfaces of general type with divisible canonical class. The construction uses branched coverings over smooth curves in pluricanonical linear systems $|n K|$. 


\section{General restrictions on the divisibility of the canonical class}

We begin by deriving a few general restrictions for symplectic 4-manifolds admitting a symplectic structure whose canonical class is divisible by an integer $d>1$.

Let $(M, \omega)$ be a closed, symplectic 4-manifold. The canonical class $K$ of the symplectic form $\omega$ is defined as

$$
K=-c_{1}(T M, J),
$$

where $J$ is an almost-complex structure compatible with $\omega$. The self-intersection number of $K$ is given by the formula $K^{2}=c_{1}^{2}(M)=2 e(M)+3 \sigma(M)$. Since the first Chern class $c_{1}(T M, J)$ is characteristic, it follows by a general property of the intersection form that $c_{1}^{2}(M) \equiv \sigma(M) \bmod 8$, and hence the number

$$
\chi_{h}(M)=\frac{1}{4}(e(M)+\sigma(M))
$$

is an integer. If $b_{1}(M)=0$, this number is equal to $\frac{1}{2}\left(1+b_{2}^{+}(M)\right)$. In particular, in this case $b_{2}^{+}(M)$ is an odd integer and $\chi_{h}(M)>0$. There is a further constraint if the manifold $M$ is spin, equivalent to the congruence $\sigma(M) \equiv 0 \bmod 16$ given by Rohlin's theorem [1952], which says that $c_{1}^{2}(M) \equiv 8 \chi_{h}(M)$ mod 16. In particular, $c_{1}^{2}(M)$ is divisible by 8 . We say that $K$ is divisible by an integer $d$ if there exists a cohomology class $A \in H^{2}(M ; \mathbb{Z})$ with $K=d A$.

Lemma 1. Let $(M, \omega)$ be a closed symplectic 4-manifold. Suppose $K$ is divisible by an integer $d$. Then $c_{1}^{2}(M)$ is divisible by $d^{2}$ if $d$ is odd and by $2 d^{2}$ if $d$ is even.

Proof. If $d$ divides $K$, we can write $K=d A$, where $A \in H^{2}(M ; \mathbb{Z})$. The equation $c_{1}^{2}(M)=K^{2}=d^{2} A^{2}$ implies that $c_{1}^{2}(M)$ is divisible by $d^{2}$ in any case. If $d$ is even, then $w_{2}(M) \equiv K \equiv 0 \bmod 2$; hence $M$ is spin and the intersection form $Q_{M}$ is even. This implies that $A^{2}$ is divisible by 2 ; hence $c_{1}^{2}(M)$ is divisible by $2 d^{2}$.

The case $c_{1}^{2}(M)=0$ is special, since there are no restrictions from this lemma; see Section 4 . For the general case of spin symplectic 4-manifolds $(d=2)$, we recover the constraint that $c_{1}^{2}$ is divisible by 8 .

Further restrictions come from the adjunction formula $2 g-2=K \cdot C+C \cdot C$, where $C$ is an embedded symplectic surface of genus $g$ oriented by the restriction of the symplectic form.

Lemma 2. Let $(M, \omega)$ be a closed symplectic 4-manifold. Suppose $K$ is divisible by an integer $d$.

- If $M$ contains a symplectic surface of genus $g$ and self-intersection 0 , then $d$ divides $2 g-2$.

- If $d \neq 1$, then $M$ is minimal. If $M$ is in addition simply connected, then it is irreducible. 
Proof. The first part follows immediately by the adjunction formula. If $M$ is not minimal, it contains a symplectically embedded sphere $S$ of self-intersection (-1). The adjunction formula can be applied and yields $K \cdot S=-1$, and hence $K$ is indivisible. The claim of irreducibility follows from [Hamilton and Kotschick 2006, Corollary 1.4].

The canonical class of a 4-manifold $M$ with $b_{2}^{+} \geq 2$ is a Seiberg-Witten basic class, that is, it has nonvanishing Seiberg-Witten invariant. This implies that only finitely many classes in $H^{2}(M ; \mathbb{Z})$ can occur as the canonical classes of symplectic structures on $M$.

Theorem 3 [Li and Liu 2001]. Let $M$ be a (smoothly) minimal closed 4-manifold with $b_{2}^{+}=1$. Then the canonical classes of all symplectic structures on $M$ are equal up to sign.

If $M$ is a Kähler surface, we can consider the canonical class of the Kähler form. Theorem 4. Suppose that $M$ is a minimal Kähler surface with $b_{2}^{+}>1$.

- If $M$ is of general type, then $\pm K_{M}$ are the only Seiberg-Witten basic classes of $M$.

- If $N$ is another minimal Kähler surface such that $b_{2}^{+}>1$ and $\phi: M \rightarrow N$ is a diffeomorphism, then $\phi^{*} K_{N}= \pm K_{M}$.

For the proofs see [Friedman and Morgan 1997; Morgan 1996; Witten 1994]. When $\phi$ is the identity diffeomorphism, the second part of this theorem has an immediate consequence:

Corollary 5. Let $M$ be a (smoothly) minimal closed 4-manifold with $b_{2}^{+}>1$. Then the canonical classes of all Kähler structures on $M$ are equal up to sign.

The corresponding statement is not true in general for the canonical classes of symplectic structures on minimal 4-manifolds with $b_{2}^{+}>1$. There exist such 4-manifolds $M$ admitting several symplectic structures whose canonical classes in $H^{2}(M ; \mathbb{Z})$ are not equal up to sign. In addition, such examples can be constructed where the canonical classes cannot be permuted by orientation-preserving self-diffeomorphisms of the manifold [McMullen and Taubes 1999; Smith 2000; Vidussi 2001], for example because they have different divisibilities as elements in integral cohomology (see the examples in Sections 8 and 9).

It is useful to define the (maximal) divisibility of the canonical class in the case that $H^{2}(M ; \mathbb{Z})$ is torsion-free.

Definition 6. Suppose $H$ is a finitely generated free abelian group. For $a \in H$, let $d(a)=\max \left\{k \in \mathbb{N}_{0} \mid\right.$ there exists a nonzero element $b \in H$ with $\left.a=k b\right\}$. 
We call $d(a)$ the divisibility of $a$ (or, for emphasis, the maximal divisibility). The divisibility of $a$ is 0 if and only $a=0$. We call $a$ indivisible if $d(a)=1$.

If $M$ is a simply connected manifold, the integral cohomology group $H^{2}(M ; \mathbb{Z})$ is torsion-free, and $K \in H^{2}(M ; \mathbb{Z})$ has a well-defined divisibility.

Proposition 7. Suppose $M$ is a simply connected closed 4-manifold that admits at least two symplectic structures whose canonical classes have different divisibilities. Then $M$ is not diffeomorphic to a complex surface.

Proof. The assumptions imply $M$ has a symplectic structure whose canonical class has divisibility $\neq 1$. By Lemma 2 , the manifold $M$ is (smoothly) minimal, and by Theorem 3, it has $b_{2}^{+}>1$. Suppose $M$ is diffeomorphic to a complex surface. The Kodaira-Enriques classification implies $M$ is diffeomorphic either to an elliptic surface $E(n)_{p, q}$ with $n \geq 2$ and $p, q$ coprime, or to a surface of general type.

Consider the elliptic surfaces $E(n)_{p, q}$ for $n \geq 2$, and denote the class of a general fibre by $F$. The Seiberg-Witten basic classes of these 4-manifolds are known [Fintushel and Stern 1997], and consist of the set of classes of the form $k f$, where $f$ denotes the indivisible class $f=F / p q$ and $k$ is an integer such that

$$
k \equiv n p q-p-q \bmod 2 \text { and }|k| \leq n p q-p-q .
$$

Suppose $\omega$ is a symplectic structure on $E(n)_{p, q}$ with canonical class $K$. By a theorem of Taubes [Taubes 1995a; Kotschick 1997], the inequality $K \cdot[\omega] \geq|c \cdot[\omega]|$ holds for any basic class $c$, with equality if and only if $K= \pm c$, and the number $K \cdot[\omega]$ is positive if $K$ is nonzero. It follows that the canonical class of any symplectic structure on $E(n)_{p, q}$ is given by $\pm(n p q-p-q) f$; hence there is only one possible divisibility. This follows for surfaces of general type by the first part of Theorem 4.

We now consider the geography question for manifolds with $c_{1}^{2}<0$. The next theorem is due to C. H. Taubes [1995b] in the case $b_{2}^{+} \geq 2$ and to A.-K. Liu [1996] in the case $b_{2}^{+}=1$.

Theorem 8. Let $M$ be a closed, symplectic 4-manifold. Suppose $M$ is minimal.

- If $b_{2}^{+}(M) \geq 2$, then $K^{2} \geq 0$.

- If $b_{2}^{+}(M)=1$ and $K^{2}<0$, then $M$ is a ruled surface, that is, an $S^{2}$-bundle over a surface (of genus $\geq 2$ ).

Since ruled surfaces over irrational curves are not simply connected, any simply connected, symplectic 4-manifold $M$ with $c_{1}^{2}(M)<0$ is not minimal. By Lemma 2, this implies that $K$ is indivisible, that is, $d(K)=1$.

Let $\left(\chi_{h}, c_{1}^{2}\right)=(n,-r)$ be a lattice point with $n, r \geq 1$, and let $M$ be a simply connected symplectic 4-manifold with these invariants. Since $M$ is not minimal, 
we can successively blow down $r(-1)$-spheres in $M$ to get a simply connected symplectic 4-manifold $N$ with invariants $\left(\chi_{h}, c_{1}^{2}\right)=(n, 0)$ such that there exists a diffeomorphism $M=N \# r \overline{\mathbb{C} P^{2}}$.

Conversely, consider the manifold $M=E(n) \# r \overline{\mathbb{C} P^{2}}$. Then $M$ is a simply connected symplectic 4-manifold with indivisible $K$. Since $\chi_{h}(E(n))=n$ and $c_{1}^{2}(E(n))=0$, this implies $\left(\chi_{h}(M), c_{1}^{2}(M)\right)=(n,-r)$. Hence the point $(n,-r)$ can be realized by a simply connected symplectic 4-manifold.

\section{The generalized fibre sum}

We next recall the definition of the generalized fibre sum from [Gompf 1995; McCarthy and Wolfson 1994] and fix some notation, used in [Hamilton 2008]. Let $M$ and $N$ be closed oriented 4-manifolds that contain embedded oriented surfaces $\Sigma_{M}$ and $\Sigma_{N}$ of genus $g$ and self-intersection 0 . We choose trivializations of the form $\Sigma_{g} \times D^{2}$ for tubular neighbourhoods of the surfaces $\Sigma_{M}$ and $\Sigma_{N}$. The generalized fibre sum $X=M \#_{\Sigma_{M}=\Sigma_{N}} N$ is then formed by deleting the interior of the tubular neighbourhoods and gluing the resulting manifolds $M^{\prime}$ and $N^{\prime}$ along their boundaries $\Sigma_{g} \times S^{1}$, using a diffeomorphism that preserves the meridians to the surfaces, given by the $S^{1}$ fibres, and reverses the orientation on them. The closed oriented 4-manifold can depend on the choice of trivializations and gluing diffeomorphism. The trivializations of the tubular neighbourhoods also determine push-offs of the central surfaces $\Sigma_{M}$ and $\Sigma_{N}$ into the boundary. Under inclusion, the push-offs determine surfaces $\Sigma_{X}$ and $\Sigma_{X}^{\prime}$ of self-intersection 0 in the 4-manifold $X$. In general, these surfaces do not represent the same homology class in $X$ but differ by a rim torus. However, if the gluing diffeomorphism is chosen so that it preserves also the $\Sigma_{g}$-fibres in $\Sigma_{g} \times S^{1}$, then the push-offs get identified to a well-defined surface $\Sigma_{X}$ in $X$.

Suppose the surfaces $\Sigma_{M}$ and $\Sigma_{N}$ represent indivisible nontorsion classes in the homology of $M$ and $N$. We can then choose surfaces $B_{M}$ and $B_{N}$ in $M$ and $N$ that intersect $\Sigma_{M}$ and $\Sigma_{N}$ at a single positive transverse point. These surfaces with a disk removed can be assumed to bound the meridians to $\Sigma_{M}$ and $\Sigma_{N}$ in the manifolds $M^{\prime}$ and $N^{\prime}$; hence they sew together to give a surface $B_{X}$ in $X$.

The second cohomology of $M$ can be split into a direct sum

$$
H^{2}(M ; \mathbb{Z}) \cong P(M) \oplus \mathbb{Z} \Sigma_{M} \oplus \mathbb{Z} B_{M},
$$

where $P(M)$ denotes the orthogonal complement to the subgroup $\mathbb{Z} \Sigma_{M} \oplus \mathbb{Z} B_{M}$ in $H^{2}(M ; \mathbb{Z})$ with respect to the intersection form $Q_{M}$. The restriction of the intersection form to the last two summands is given by

$$
\left(\begin{array}{cc}
0 & 1 \\
1 & B_{M}^{2}
\end{array}\right)
$$


This form is unimodular; hence the restriction of the intersection form to $P(M)$ (modulo torsion) is unimodular as well. There exists a similar decomposition for the second cohomology of $N$.

Theorem 9. Suppose that the integral cohomology of $M, N$ and $X$ is torsion-free and the surfaces $\Sigma_{M}$ and $\Sigma_{N}$ represent indivisible classes. If rim tori do not exist in the fibre sum $X=M \# \Sigma_{M}=\Sigma_{N} N$, then the second cohomology of $X$ splits as a direct sum

$$
H^{2}(X ; \mathbb{Z}) \cong P(X) \oplus \mathbb{Z} \Sigma_{X} \oplus \mathbb{Z} B_{X}, \quad \text { where } P(X) \cong P(M) \oplus P(N) .
$$

The restriction of the intersection form $Q_{X}$ to $P(X)$ is the direct sum of the restrictions of $Q_{M}$ and $Q_{N}$, and the restriction to $\mathbb{Z} \Sigma_{X} \oplus \mathbb{Z} B_{X}$ is of the form

$$
\left(\begin{array}{cc}
0 & 1 \\
1 & B_{M}^{2}+B_{N}^{2}
\end{array}\right)
$$

A proof for this theorem can be found in [Hamilton 2008, Section V.3.5]. It implies that there exist monomorphisms of abelian groups of both $H^{2}(M ; \mathbb{Z})$ and $H^{2}(N ; \mathbb{Z})$ into $H^{2}(X ; \mathbb{Z})$ given by

$$
\Sigma_{M} \mapsto \Sigma_{X}, \quad B_{M} \mapsto B_{X}, \quad \operatorname{Id}: P(M) \rightarrow P(M),
$$

and similarly for $N$. The monomorphisms do not preserve the intersection form if $B_{M}^{2}$ or $B_{N}^{2}$ differ from $B_{X}^{2}$. The next lemma can be useful in checking the conditions for Theorem 9; its proof follows from [Hamilton 2008, Sections V.2 and V.3].

Lemma 10. Let $X=M \# \Sigma_{M}=\Sigma_{N} N$ be a generalized fibre sum along embedded surfaces of self-intersection 0. Suppose that the map on integral first homology induced by one of the embeddings, say $\Sigma_{N} \rightarrow N$, is an isomorphism. Then rim tori do not exist in $X$. If in addition one of the surfaces represents an indivisible homology class, then $H_{1}(X ; \mathbb{Z}) \cong H_{1}(M ; \mathbb{Z})$.

Suppose $M$ and $N$ are symplectic 4-manifolds and $\Sigma_{M}$ and $\Sigma_{N}$ symplectically embedded. We orient both surfaces by the restriction of the symplectic forms. Then the generalized fibre sum $X$ also admits a symplectic structure. The canonical class $K_{X}$ can be calculated as follows:

Theorem 11. Under the assumptions of Theorem 9 and the embeddings of the cohomology of $M$ and $N$ into the cohomology of $X$ given by Equation (1), we have

$$
K_{X}=K_{M}+K_{N}-(2 g-2) B_{X}+2 \Sigma_{X} .
$$

A proof can be found in [Hamilton 2008, Section V.5]. The formula for $g=1$ has been proved in [Smith 2000] and a related formula for arbitrary $g$ can be found in [Ionel and Parker 2004]. 


\section{The knot surgery construction}

We will frequently use the following construction due to Fintushel and Stern [1998]. Let $K$ be a knot in $S^{3}$, and denote a tubular neighbourhood of $K$ by $v K \cong S^{1} \times D^{2}$. Let $m$ be a fibre of the circle bundle $\partial v K \rightarrow K$, and use an oriented Seifert surface for $K$ to define a section $l: K \rightarrow \partial v K$. The circles $m$ and $l$ are called the meridian and the longitude of $K$. Let $M_{K}$ be the closed 3-manifold obtained by 0-Dehn surgery on $K$. The manifold $M_{K}$ is constructed as follows: Consider $S^{3} \backslash \operatorname{int} v K$ and let $f: \partial\left(S^{1} \times D^{2}\right) \rightarrow \partial\left(S^{3} \backslash\right.$ int $\left.\nu K\right)$ be a diffeomorphism that maps the circle $\partial D^{2}$ onto $l$. Then one defines

$$
M_{K}=\left(S^{3} \backslash \operatorname{int} v K\right) \cup_{f}\left(S^{1} \times D^{2}\right) .
$$

The manifold $M_{K}$ is determined by this construction uniquely up to diffeomorphism. One can show that it has the same integral homology as $S^{2} \times S^{1}$. The meridian $m$, which bounds the fibre in the normal bundle to $K$ in $S^{3}$, becomes nonzero in the homology of $M_{K}$ and defines a generator for $H_{1}\left(M_{K} ; \mathbb{Z}\right)$. The longitude $l$ is null-homotopic in $M_{K}$, since it bounds one of the $D^{2}$ fibres glued in. This disk fibre together with the Seifert surface of $K$ determine a closed, oriented surface $B_{K}$ in $M_{K}$ that intersects $m$ once and generates $H_{2}\left(M_{K} ; \mathbb{Z}\right)$.

We consider the closed, oriented 4-manifold $M_{K} \times S^{1}$. It contains an embedded torus $T_{K}=m \times S^{1}$ of self-intersection 0 , which has a framing coming from a canonical framing of $m$. Let $X$ be an arbitrary closed, oriented 4-manifold, which contains an embedded torus $T_{X}$ of self-intersection 0 , representing an indivisible homology class. Then the result of knot surgery on $X$ is given by the generalized fibre sum $X_{K}=X \#_{T_{X}=T_{K}}\left(M_{K} \times S^{1}\right)$. Here we have implicitly chosen a trivialization of the form $T^{2} \times D^{2}$ for the tubular neighbourhood of the torus $T_{X}$. We choose a gluing diffeomorphism that preserves both the $T^{2}$ factor and the $S^{1}$ factor on the boundaries of the tubular neighbourhoods and reverses orientation on the $S^{1}$ factor (the smooth 4-manifold $X_{K}$ might depend on the choice of the framing for $T_{X}$ ). The embedded torus of self-intersection 0 in $X_{K}$, defined by identifying the push-offs, is denoted by $T_{X_{K}}$.

The closed surface $B_{K}$ in the 3-manifold $M_{K}$ determines under inclusion a closed surface in the 4-manifold $M_{K} \times S^{1}$, denoted by the same symbol. It intersects the torus $T_{K}$ at a single transverse point. We also choose a surface $B_{X}$ in $X$ intersecting $T_{X}$ transversely and geometrically once. Both surfaces sew together to form a surface $B_{X_{K}}$ in $X_{K}$ that intersects the torus $T_{X_{K}}$ at a single transverse point.

We assume the cohomology of $X$ is torsion-free. By [Fintushel and Stern 1998], it is known that there exists an isomorphism

$$
H^{2}(X ; \mathbb{Z}) \cong H^{2}\left(X_{K} ; \mathbb{Z}\right)
$$


preserving intersection forms. In the notation of Section 2 this follows because $H^{2}\left(M_{K} \times S^{1} ; \mathbb{Z}\right) \cong \mathbb{Z} T_{K} \oplus \mathbb{Z} B_{K}$ and hence $P\left(M_{K} \times S^{1}\right)=0$. In addition, the self-intersection number of $B_{X_{K}}$ is equal to the self-intersection number of $B_{X}$, because the class $B_{K}$ has zero self-intersection (it can be moved away in the $S^{1}$ direction). The claim then follows from Theorem 9 and Lemma 10.

In particular, assume that both $X$ and $X^{\prime}=X \backslash T_{X}$ are simply connected. Since the fundamental group of $M_{K} \times S^{1}$ is normally generated by the image of the fundamental group of $T_{K}$ under inclusion, it follows that $X_{K}$ is again simply connected; hence by Freedman's theorem [1982], the manifolds $X$ and $X_{K}$ are homeomorphic. However, one can show with Seiberg-Witten theory that $X$ and $X_{K}$ are in many cases not diffeomorphic [Fintushel and Stern 1998].

Suppose that $K$ is a fibred knot, that is, there exists a fibration

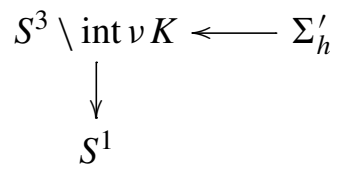

over the circle, where the fibres $\Sigma_{h}^{\prime}$ are punctured surfaces of genus $h$ forming Seifert surfaces for $K$. Then $M_{K}$ is fibred by closed surfaces $B_{K}$ of genus $h$. This induces a fibre bundle

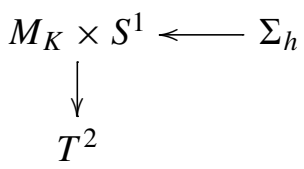

and the torus $T_{K}=m \times S^{1}$ is a section of this bundle. By a theorem of Thurston [1976] the manifold $M_{K} \times S^{1}$ admits a symplectic form such that $T_{K}$ and the fibres are symplectic. This construction can be used to do symplectic generalized fibre sums along $T_{K}$ if the manifold $X$ is symplectic and the torus $T_{X}$ symplectically embedded. The canonical class of $M_{K} \times S^{1}$ can be calculated by the adjunction formula, because the fibres $B_{K}$ and the torus $T_{K}$ are symplectic surfaces and form a basis of $H_{2}\left(M_{K} \times S^{1} ; \mathbb{Z}\right)$. The result is $K_{M_{K} \times S^{1}}=(2 h-2) T_{K}$. According to [Fintushel and Stern 1998], the canonical class of the symplectic 4-manifold $X_{K}$ is then given by

$$
K_{X_{K}}=K_{X}+2 h T_{X}
$$

See also Theorem 11.

\section{Symplectic 4-manifolds with $c_{1}^{2}=0$}

Definition 12. A closed, simply connected 4-manifold $M$ is called a homotopy elliptic surface if $M$ is homeomorphic to a relatively minimal, simply connected 
elliptic surface, that is, to a complex surface of the form $E(n)_{p, q}$ with $p, q$ coprime and $n \geq 1$.

For details on the surfaces $E(n)_{p, q}$, see [Gompf and Stipsicz 1999, Section 3.3]. By definition, homotopy elliptic surfaces $M$ are simply connected with invariants

$$
c_{1}^{2}(M)=0, \quad e(M)=12 n, \quad \sigma(M)=-8 n .
$$

The integer $n$ is equal to $\chi_{h}(M)$. In particular, $K^{2}=0$ for symplectic homotopy elliptic surfaces. There is a converse:

Lemma 13. Let $M$ be a closed, simply connected, symplectic 4-manifold with $K^{2}=0$. Then $M$ is a homotopy elliptic surface.

Proof. Since $M$ is almost complex, $\chi_{h}(M)$ is an integer. The Noether formula

$$
\chi_{h}(M)=\frac{1}{12}\left(K^{2}+e(M)\right)=\frac{1}{12} e(M)
$$

implies that $e(M)$ is divisible by 12 ; hence $e(M)=12 k$ for some $k>0$. Together with the equation

$$
0=K^{2}=2 e(M)+3 \sigma(M),
$$

it follows that $\sigma(M)=-8 k$. Suppose that $M$ is nonspin. If $k$ is odd, then $M$ has the same Euler characteristic, signature and type as $E(k)$. If $k$ is even, then $M$ has the same Euler characteristic, signature and type as the nonspin manifold $E(k)_{2}$. Since $M$ is simply connected, $M$ is homeomorphic to the corresponding elliptic surface by Freedman's theorem [1982].

Suppose that $M$ is spin. Then the signature is divisible by 16 due to Rohlin's theorem. Hence the integer $k$ above has to be even. Then $M$ has the same Euler characteristic, signature and type as the spin manifold $E(k)$. Again by Freedman's theorem, the 4-manifold $M$ is homeomorphic to $E(k)$.

Lemma 14. Suppose that $M$ is a symplectic homotopy elliptic surface such that the divisibility of $K$ is even. Then $\chi_{h}(M)$ is even.

Proof. The assumption implies that $M$ is spin. The Noether formula then shows that $\chi_{h}(M)$ is even, since $K^{2}=0$ and $\sigma(M)$ is divisible by 16 .

The next theorem shows that this is the only restriction on the divisibility of the canonical class $K$ for symplectic homotopy elliptic surfaces.

Theorem 15 (homotopy elliptic surfaces). Let $n$ and $d$ be positive integers. If $n$ is odd, assume that $d$ is odd also. Then there exists a symplectic homotopy elliptic surface $(M, \omega)$ with $\chi_{h}(M)=n$ whose canonical class $K$ has divisibility $d$.

Note that there is no constraint on $d$ if $n$ is even. 
Proof. If $n$ is equal to 1 or 2, the symplectic manifold can be realized as an elliptic surface. The canonical class of an elliptic surface $E(n)_{p, q}$ with $p, q$ coprime is given by $K=(n p q-p-q) f$, where $f$ is indivisible and $F=p q f$ denotes the class of a general fibre. For $n=1$ and $d$ odd, we can take the surface $E(1)_{d+2,2}$, since

$$
(d+2) 2-(d+2)-2=d .
$$

For $n=2$ and $d$ arbitrary, we can take $E(2)_{d+1}=E(2)_{d+1,1}$, since

$$
2(d+1)-(d+1)-1=d .
$$

We now consider the case $n \geq 1$ in general and separate the proof into several cases.

Case: $d=2 k$ and $n=2 m$ are both even with $k, m \geq 1$. Consider the elliptic surface $E(n)$. It contains a general fibre $F$ that is an embedded symplectic torus of self-intersection 0 . It also contains a rim torus $R$ that arises from a decomposition of $E(n)$ as a fibre sum $E(n)=E(n-1) \#_{F} E(1)$; see [Gompf and Mrowka 1993] and Example 30. The rim torus $R$ has self-intersection 0 and there exists a dual (Lagrangian) 2-sphere $S$ with intersection $R S=1$. We can assume that $R$ and $S$ are disjoint from the fibre $F$. The rim torus is in a natural way Lagrangian. By a perturbation of the symplectic form, we can assume that it becomes symplectic. We give $R$ the orientation induced by the symplectic form. The proof consists in doing knot surgery along the fibre $F$ and the rim torus $R$.

Let $K_{1}$ be a fibred knot of genus $g_{1}=m(k-1)+1$. We do knot surgery along $F$ with the knot $K_{1}$ to get a new symplectic 4-manifold $M_{1}$. The elliptic fibration $E(n) \rightarrow \mathbb{C} P^{l}$ has a section showing that the meridian of $F$, which is the $S^{1}$ fibre of $\partial v F \rightarrow F$, bounds a disk in $E(n) \backslash$ int $v F$. This implies that the complement of $F$ in $E(n)$ is simply connected; hence the manifold $M_{1}$ is again simply connected. By the knot surgery construction the manifold $M_{1}$ is homeomorphic to $E(n)$. The canonical class is given by formula (3):

$$
K_{M_{1}}=(n-2) F+2 g_{1} F=(2 m-2+2 m k-2 m+2) F=2 m k F .
$$

Here we have identified the cohomology of $M_{1}$ and $E(n)$ under the isomorphism in Equation (2). The rim torus $R$ still exists as an embedded oriented symplectic torus in $M_{1}$ with a dual 2-sphere $S$ because we can assume that the knot surgery takes place in a small neighbourhood of $F$ disjoint from $R$ and $S$. In particular, the complement of $R$ in $M_{1}$ is simply connected. Let $K_{2}$ be a fibred knot of genus $g_{2}=k$, and let $M$ be the result of knot surgery on $M_{1}$ along $R$. Then $M$ is a simply connected symplectic 4-manifold homeomorphic to $E(n)$. The canonical class of $M$ is given by $K=2 m k F+2 k R$. 
The cohomology class $K$ is divisible by $2 k$. The sphere $S$ sews together with a Seifert surface for the knot $K_{2}$ to give a surface $C$ in $M$ with $C \cdot R=1$ and $C \cdot F=0$; hence $C \cdot K=2 k$. Therefore the divisibility of $K$ is precisely $d=2 k$.

Case: $d=2 k+1$ and $n=2 m+1$ are both odd with $k \geq 0$ and $m \geq 1$. We consider the elliptic surface $E(n)$ and do a similar construction. Let $K_{1}$ be a fibred knot of genus $g_{1}=2 k m+k+1$, and do knot surgery along $F$ as above. We get a simply connected symplectic 4-manifold $M_{1}$ with canonical class

$$
\begin{aligned}
& K_{M_{1}}=(n-2) F+2 g_{1} F \\
&=(2 m+1-2+4 k m+2 k+2) F=(4 k m+2 k+2 m+1) F \\
&=(2 m+1)(2 k+1) F .
\end{aligned}
$$

Next we consider a fibred knot $K_{2}$ of genus $g_{2}=2 k+1$ and do knot surgery along the rim torus $R$. The result is a simply connected symplectic 4-manifold $M$ homeomorphic to $E(n)$ with canonical class $K=(2 m+1)(2 k+1) F+2(2 k+1) R$, which is divisible by $(2 k+1)$. The same argument as above shows that there is a surface $C$ in $M$ with $C \cdot K=2(2 k+1)$. We claim that the divisibility of $K$ is precisely $(2 k+1)$ : This follows because $M$ is still homeomorphic to $E(n)$ by the knot surgery construction. Since $n$ is odd, the manifold $M$ is not spin and hence 2 does not divide $K$. (An explicit surface with odd intersection number can be constructed from a section of $E(n)$ and a Seifert surface for the knot $K_{1}$. This surface has self-intersection number $-n$ and intersection number $(2 m+1)(2 k+1)$ with $K$.)

To cover the remaining case $m=0$ (corresponding to $n=1$ ), we can do knot surgery on the elliptic surface $E(1)$ along a general fibre $F$ with a knot $K_{1}$ of genus $g_{1}=k+1$. The resulting manifold $M_{1}$ has canonical class

$$
K_{M_{1}}=-F+(2 k+2) F=(2 k+1) F .
$$

Case: $d=2 k+1$ is odd and $n=2 m$ is even with $k \geq 0$ and $m \geq 1$. We consider the elliptic surface $E(n)$ and first perform a logarithmic transformation along $F$ of index 2. Let $f$ denote the multiple fibre such that $F$ is homologous to $2 f$. There exists a 2-sphere in $E(n)_{2}$ that intersects $f$ at a single point (for a proof see Lemma 16). In particular, the complement of $f$ in $E(n)_{2}$ is simply connected. The canonical class of $E(n)_{2}=E(n)_{2,1}$ is given by $K=(2 n-3) f$. We can assume that the torus $f$ is symplectic (for example, by considering the logarithmic transformation to be done on the complex algebraic surface $E(n)$, resulting in the complex algebraic surface $\left.E(n)_{2}\right)$. Let $K_{1}$ be a fibred knot of genus $g_{1}=$ $4 k m+k+2$, and do knot surgery along $f$ with $K_{1}$ as above. The result is a simply connected symplectic 4-manifold homeomorphic to $E(n)_{2}$. The canonical class is 
given by

$$
\begin{aligned}
K_{M_{1}}=(2 n-3) f+2 g_{1} f & \\
=(4 m-3+8 k m+2 k+4) f & =(8 k m+4 m+2 k+1) f \\
& =(4 m+1)(2 k+1) f .
\end{aligned}
$$

We now consider a fibred knot $K_{2}$ of genus $g_{2}=2 k+1$ and do knot surgery along the rim torus $R$. We get a simply connected symplectic 4-manifold $M$ homeomorphic to $E(n)_{2}$ with canonical class $K=(4 m+1)(2 k+1) f+2(2 k+1) R$. A similar argument as above shows that the divisibility of $K$ is $d=2 k+1$.

Lemma 16. Let $p \geq 1$ be an integer, and let $f$ be the multiple fibre in $E(n)_{p}$. Then there exists a sphere in $E(n)_{p}$ that intersects $f$ transversely at one point.

Proof. We can think of the logarithmic transformation as gluing $T^{2} \times D^{2}$ into $E(n) \backslash$ int $v F$ by a certain diffeomorphism $\phi: T^{2} \times S^{1} \rightarrow \partial v F$. The fibre $f$ corresponds to $T^{2} \times\{0\}$. Consider a disk of the form $\{*\} \times D^{2}$. It intersects $f$ once, and its boundary maps under $\phi$ to a certain simple closed curve on $\partial v F$. Since $E(n) \backslash \operatorname{int} v F$ is simply connected, this curve bounds a disk in $E(n) \backslash \operatorname{int} v F$. The union of this disk and the disk $\{*\} \times D^{2}$ is a sphere in $E(n)_{p}$ that intersects $f$ transversely once.

Remark 17. Under the assumptions of Theorem 15, it is possible to construct infinitely many homeomorphic but pairwise nondiffeomorphic symplectic homotopy elliptic surfaces $\left(M_{r}\right)_{r \in \mathbb{N}}$ with $\chi_{h}\left(M_{r}\right)=n$, whose canonical classes all have divisibility equal to $d$. This follows because we can vary in each case the knot $K_{1}$ and its genus $g_{1}$ without changing the divisibility of the canonical class. The claim then follows by the formula for the Seiberg-Witten invariants of knot surgery manifolds [Fintushel and Stern 1998].

\section{Generalized knot surgery}

Symplectic manifolds with $c_{1}^{2}>0$ and divisible canonical class can be constructed with a version of knot surgery for higher genus surfaces described in [Fintushel and Stern 2004]. Let $K=K_{h}$ denote the $(2 h+1,-2)$-torus knot, which is a fibred knot of genus $h$. Consider the manifold $M_{K} \times S^{1}$ from the knot surgery construction of Section 3. This manifold has the structure of a $\Sigma_{h}$-bundle over $T^{2}$ :

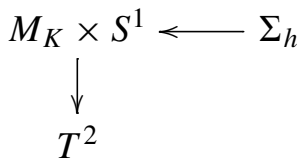

We denote a fibre of this bundle by $\Sigma_{F}$. The fibration defines a trivialization of the normal bundle $v \Sigma_{F}$. We form $g$ consecutive generalized fibre sums along the 
fibres $\Sigma_{F}$ to get

$$
Y_{g, h}=\left(M_{K} \times S^{1}\right) \#_{\Sigma_{F}=\Sigma_{F}} \# \cdots \# \Sigma_{F}=\Sigma_{F}\left(M_{K} \times S^{1}\right) .
$$

We choose the gluing diffeomorphism so that it identifies the $\Sigma_{h}$ fibres in the boundary of the tubular neighbourhoods. This implies that $Y_{g, h}$ is a $\Sigma_{h}$-bundle over $\Sigma_{g}$ :

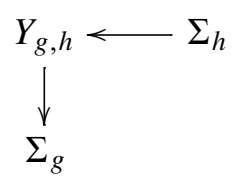

We denote the fibre again by $\Sigma_{F}$. The fibre bundle has a section $\Sigma_{S}$ sewed together from $g$ torus sections of $M_{K} \times S^{1}$. Since the knot $K$ is a fibred knot, the manifold $M_{K} \times S^{1}$ admits a symplectic structure such that the fibre and the section are symplectic. By the Gompf construction this is then also true for $Y_{g, h}$.

The invariants of the 4-manifold $Y_{g, h}$ can be calculated by standard formulas [Park 2002, Lemma 2.4]:

$$
c_{1}^{2}\left(Y_{g, h}\right)=8(g-1)(h-1), \quad e\left(Y_{g, h}\right)=4(g-1)(h-1), \quad \sigma\left(Y_{g, h}\right)=0 .
$$

By induction on $g$, one can show that the fundamental group $\pi_{1}\left(Y_{g, h}\right)$ is normally generated by the image of $\pi_{1}\left(\Sigma_{S}\right)$ under inclusion [Fintushel and Stern 2004, Proposition 2]. This fact, together with the exact sequence

$$
H_{1}\left(\Sigma_{F}\right) \rightarrow H_{1}\left(Y_{g, h}\right) \rightarrow H_{1}\left(\Sigma_{g}\right) \rightarrow 0
$$

coming from the long exact homotopy sequence for the fibration $\Sigma_{F} \rightarrow Y_{g, h} \rightarrow \Sigma_{g}$ by abelianization, shows that the inclusion $\Sigma_{S} \rightarrow Y_{g, h}$ induces an isomorphism on $H_{1}$ and the inclusion $\Sigma_{F} \rightarrow Y_{g, h}$ induces the zero map. In particular, the homology group $H_{1}\left(Y_{g, h} ; \mathbb{Z}\right)$ is free abelian of rank $b_{1}\left(Y_{g, h}\right)=g b_{1}\left(M_{K} \times S^{1}\right)=2 g$. This implies with the formula for the Euler characteristic above that

$$
b_{2}\left(Y_{g, h}\right)=4 h(g-1)+2 .
$$

The summand $4 h(g-1)$ results from $2 h$ split classes (or vanishing classes) together with $2 h$ dual rim tori that are created in each fibre sum. The split classes are formed as follows: In each fibre sum, the interior of a tubular neighbourhood $v \Sigma_{F}$ of a fibre on each side of the sum is deleted and the boundaries $\partial v \Sigma_{F}$ glued together such that the fibres inside the boundary get identified pairwise. Since the inclusion of the fibre $\Sigma_{F}$ into $M_{K} \times S^{1}$ induces the zero map on first homology, the $2 h$ generators of $H_{1}\left(\Sigma_{h}\right)$, where $\Sigma_{h}$ is considered as a fibre in $\partial v \Sigma_{F}$, bound surfaces in $M_{K} \times S^{1}$ minus the interior of the tubular neighbourhood $v \Sigma_{F}$. The split classes arise from sewing together surfaces bounding corresponding generators on each side of the fibre sum. Fintushel and Stern show that in the case above there exists a basis for 
the group of split classes consisting of $2 h(g-1)$ disjoint surfaces of genus 2 and self-intersection 2 . This implies

$$
H^{2}\left(Y_{g, h} ; \mathbb{Z}\right)=2 h(g-1)\left(\begin{array}{ll}
2 & 1 \\
1 & 0
\end{array}\right) \oplus\left(\begin{array}{ll}
0 & 1 \\
1 & 0
\end{array}\right),
$$

where the last summand is the intersection form on $\left(\mathbb{Z} \Sigma_{S} \oplus \mathbb{Z} \Sigma_{F}\right)$. They also show that the canonical class of $Y_{g, h}$ is given by $K_{Y}=(2 h-2) \Sigma_{S}+(2 g-2) \Sigma_{F}$, where $\Sigma_{S}$ and $\Sigma_{F}$ are oriented by the symplectic form.

Let $M$ be a closed symplectic 4-manifold that contains a symplectic surface $\Sigma_{M}$ of genus $g$ and self-intersection 0 , oriented by the symplectic form and representing an indivisible homology class. We can then form the symplectic generalized fibre sum $X=M \#_{\Sigma_{M}=\Sigma_{S}} Y_{g, h}$. If the manifolds $M$ and $M \backslash \Sigma_{M}$ are simply connected, then $X$ is again simply connected because the fundamental group of $Y_{g, h}$ is normally generated by the image of $\pi_{1}\left(\Sigma_{S}\right)$. Since the inclusion of the surface $\Sigma_{S}$ in $Y_{g, h}$ induces an isomorphism on first homology, it follows by Theorem 9 and Lemma 10 that

$$
H^{2}(X ; \mathbb{Z})=P(M) \oplus P\left(Y_{g, h}\right) \oplus\left(\mathbb{Z} B_{X} \oplus \mathbb{Z} \Sigma_{X}\right) .
$$

The surface $B_{X}$ is sewed together from a surface $B_{M}$ in $M$ with $B_{M} \Sigma_{M}=1$ and the fibre $\Sigma_{F}$ in the manifold $Y_{g, h}$. Since $\Sigma_{F}^{2}=0$, the embedding $H^{2}(M ; \mathbb{Z}) \rightarrow$ $H^{2}(X ; \mathbb{Z})$ given by Equation (1) preserves the intersection form. Therefore we can write

$$
H^{2}(X ; \mathbb{Z})=H^{2}(M ; \mathbb{Z}) \oplus P\left(Y_{g, h}\right)
$$

with intersection form

$$
Q_{X}=Q_{M} \oplus 2 h(g-1)\left(\begin{array}{ll}
2 & 1 \\
1 & 0
\end{array}\right)
$$

The invariants of $X$ are given by

$$
c_{1}^{2}(X)=c_{1}^{2}(M)+8 h(g-1), \quad e(X)=e(M)+4 h(g-1), \quad \sigma(X)=\sigma(M) .
$$

The canonical class of $X$ can be calculated by Theorem 11 to be

$$
K_{X}=K_{M}+2 h \Sigma_{M},
$$

where the isomorphism in (4) is understood (this formula follows also from the calculation of Seiberg-Witten invariants in [Fintushel and Stern 2004]). Equation (5) is a generalization of Equation (3). In particular, we get:

Proposition 18. Suppose that $M$ is a closed, symplectic 4-manifold that contains a symplectic surface $\Sigma_{M}$ of genus $g>1$ and self-intersection 0 . Suppose that $\pi_{1}(M)=\pi_{1}\left(M \backslash \Sigma_{M}\right)=1$ and that the canonical class of $M$ is divisible by $d$. 
- If $d$ is odd, there exists for every integer $t \geq 1$ a simply connected symplectic 4-manifold $X$ with invariants

$$
c_{1}^{2}(X)=c_{1}^{2}(M)+8 t d(g-1), \quad e(X)=e(M)+4 t d(g-1), \quad \sigma(X)=\sigma(M)
$$

and canonical class divisible by $d$.

- If $d$ is even, there exists for every integer $t \geq 1$ a simply connected symplectic 4-manifold $X$ with invariants

$$
\begin{aligned}
& c_{1}^{2}(X)=c_{1}^{2}(M)+4 t d(g-1), \quad e(X)=e(M)+2 t d(g-1), \quad \sigma(X)=\sigma(M) \\
& \quad \text { and canonical class divisible by } d .
\end{aligned}
$$

This follows from the construction above by taking the genus of the torus knot $h=t d$ if $d$ is odd and $h=\frac{1}{2} t d$ if $d$ is even. Hence if a symplectic surface $\Sigma_{M}$ of genus $g>1$ and self-intersection 0 exists in $M$, we can raise $c_{1}^{2}$ without changing the signature or the divisibility of the canonical class.

\section{Spin symplectic 4-manifolds with $c_{1}^{2}>0$ and negative signature}

We can apply the construction from Section 5 to the symplectic homotopy elliptic surfaces constructed in Theorem 15. In this section we consider the case of even divisibility $d$ and in the following section the case of odd $d$.

Recall that in the first case in the proof of Theorem 15, we constructed a simply connected symplectic 4-manifold $M$ from the elliptic surface $E(2 m)$ by doing knot surgery along a general fibre $F$ with a fibred knot $K_{1}$ of genus $g_{1}=(k-1) m+1$ and a further knot surgery along a rim torus $R$ with a fibred knot $K_{2}$ of genus $g_{2}=k$. Here $2 m \geq 2$ and $d=2 k \geq 2$ are arbitrary even integers. The canonical class is given by

$$
K_{M}=2 m k F+2 k R=m d F+d R .
$$

The manifold $M$ is still homeomorphic to $E(2 m)$. There is an embedded 2-sphere $S$ in $E(2 m)$ of self-intersection -2 that intersects the rim torus $R$ once. The sphere $S$ is naturally Lagrangian [Auroux et al. 2005]. We can assume that $S$ is disjoint from the fibre $F$ and by a perturbation of the symplectic structure on $E(2 m)$ that the regular fibre $F$, the rim torus $R$ and the dual 2-sphere $S$ are all symplectic and the symplectic form induces a positive volume form on each of them; see the proofs of [Fintushel and Stern 2001, Lemma 2.1] and [Vidussi 2007, Proposition 3.2].

The 2-sphere $S$ minus a disk sews together with a Seifert surface for $K_{2}$ to give a symplectic surface $C$ in $M$ of genus $k$ and self-intersection -2 that intersects the rim torus $R$ once. By smoothing the double point we get a symplectic surface $\Sigma_{M}$ in $M$ of genus $g=k+1$ and self-intersection 0 that represents $C+R$.

The complement of $\Sigma_{M}$ in $M$ is simply connected, since we can assume $R \cup S$ in the elliptic surface $E(2 m)$ is contained in an embedded nucleus $N(2)$; see 
[Gompf and Mrowka 1993; Gompf and Stipsicz 1999] and Example 30. Inside the nucleus $N(2)$ there exists a cusp that is homologous to $R$ and disjoint from it. The cusp is still contained in $M$ and intersects the surface $\Sigma_{M}$ once. Since $M$ is simply connected and the cusp homeomorphic to $S^{2}$, the claim $\pi_{1}\left(M \backslash \Sigma_{M}\right)=1$ follows. ${ }^{1}$

Let $t \geq 1$ be an arbitrary integer, and let $K_{3}$ be the $(2 h+1,-2)$-torus knot of genus $h=t k$. Consider the generalized fibre sum $X=M \#_{\Sigma_{M}=\Sigma_{S}} Y_{g, h}$. Then $X$ is a simply connected symplectic 4-manifold with invariants

$$
c_{1}^{2}(X)=8 t k^{2}=2 t d^{2}, \quad e(X)=24 m+4 t k^{2}=24 m+t d^{2}, \quad \sigma(X)=-16 m .
$$

The canonical class is given by

$$
K_{X}=K_{M}+2 t k \Sigma_{M}=d\left(m F+R+t \Sigma_{M}\right) .
$$

Hence $K_{X}$ has divisibility $d$, since the class $m F+R+t \Sigma_{M}$ has intersection 1 with $\Sigma_{M}$. Therefore:

Theorem 19. Let $d \geq 2$ be an even integer. Then for every pair $m, t$ of positive integers, there exists a simply connected closed spin symplectic 4-manifold X with invariants

$$
c_{1}^{2}(X)=2 t d^{2}, \quad e(X)=t d^{2}+24 m, \quad \sigma(X)=-16 m,
$$

such that the canonical class $K_{X}$ has divisibility d.

Note that this solves by Lemma 1 and Rohlin's theorem the existence question for simply connected 4-manifolds with canonical class divisible by an even integer and negative signature. In particular (for $d=2$ ), every possible lattice point with $c_{1}^{2}>0$ and $\sigma<0$ can be realized by a simply connected spin symplectic 4-manifold with this construction; the existence of such 4-manifolds has been proved similarly in [Park and Szabó 2000].

Example 20 (spin homotopy Horikawa surfaces). To identify the homeomorphism type of some of the manifolds in Theorem 19 , let $d=2 k$; hence

$$
c_{1}^{2}(X)=8 t k^{2} \quad \text { and } \quad \chi_{h}(X)=t k^{2}+2 m .
$$

We consider the case when the invariants are on the Noether line $c_{1}^{2}=2 \chi_{h}-6$. This happens if and only if $6 t k^{2}=4 m-6$ and hence $2 m=3 t k^{2}+3$, which has a solution if and only if both $t$ and $k$ are odd. Hence for every pair $t, k \geq 1$ of odd integers, there exists a simply connected symplectic 4-manifold $X$ with invariants

$$
c_{1}^{2}(X)=8 t k^{2} \quad \text { and } \quad \chi_{h}(X)=4 t k^{2}+3
$$

such that the divisibility of $K_{X}$ is $2 k$.

\footnotetext{
${ }^{1}$ This argument is similar to the argument showing that the complement of a section in $E(n)$ is simply connected; see [Gompf 1995, Example 5.2].
} 
By a construction of Horikawa [1976a], there exists for every odd integer $r \geq 1$ a simply connected spin complex algebraic surface $M$ on the Noether line with invariants

$$
c_{1}^{2}(M)=8 r \quad \text { and } \quad \chi_{h}(M)=4 r+3 .
$$

See also [Gompf and Stipsicz 1999, Theorem 7.4.20] where this surface is called $U(3, r+1)$.

By Freedman's theorem [1982] the symplectic 4-manifolds $X$ constructed above for odd parameters $t$ and $k$ are homeomorphic to spin Horikawa surfaces with $r=t k^{2}$. If $k>1$ and $t$ is arbitrary, the canonical class of $X$ has divisibility $2 k>2$. In this case the manifold $X$ cannot be diffeomorphic to a Horikawa surface: It is known by [Horikawa 1976a] that all Horikawa surfaces $M$ have a fibration in genus 2 curves; hence by Lemma 2 the divisibility of $K_{M}$ is at most 2 and in the spin case is equal to 2. Since Horikawa surfaces are minimal complex surfaces of general type, the claim follows by Proposition 7 .

\section{Nonspin symplectic 4-manifolds with $c_{1}^{2}>0$ and negative signature}

We now we construct some families of simply connected symplectic 4-manifolds with $c_{1}^{2}>0$ such that the divisibility of $K$ is a given odd integer $d>1$. However, we do not have a complete existence result as in Theorem 19.

We consider the case that the canonical class $K_{X}$ is divisible by an odd integer $d$ and the signature $\sigma(X)$ is divisible by 8 .

Lemma 21. Let $X$ be a closed simply connected symplectic 4-manifold such that $K_{X}$ is divisible by an odd integer $d \geq 1$ and $\sigma(X)$ is divisible by 8 . Then $c_{1}^{2}(X)$ is divisible by $8 d^{2}$.

Proof. Suppose that $\sigma(X)=8 m$ for some integer $m \in \mathbb{Z}$. Then $b_{2}^{-}(X)=b_{2}^{+}(X)-8 m$ hence $b_{2}(X)=2 b_{2}^{+}(X)-8 m$. This implies $e(X)=2 b_{2}^{+}(X)+2-8 m$. Since $X$ is symplectic, the integer $b_{2}^{+}(X)$ is odd, so we can write $b_{2}^{+}(X)=2 k+1$ for some $k \geq 0$. This implies $e(X)=4 k+4-8 m$; hence $e(X)$ is divisible by 4 . The equation $c_{1}^{2}(X)=2 e(X)+3 \sigma(X)$ shows that $c_{1}^{2}(X)$ is divisible by 8 . Since $c_{1}^{2}(X)$ is also divisible by the odd integer $d^{2}$, the claim follows.

The following theorem covers the case that $K_{X}$ has odd divisibility and the signature is negative, divisible by 8 and no greater than -16 :

Theorem 22. Let $d \geq 1$ be an odd integer. Then for every pair $n, t$ of positive integers with $n \geq 2$, there is a simply connected closed nonspin symplectic 4-manifold $X$ with invariants

$$
c_{1}^{2}(X)=8 t d^{2}, \quad e(X)=4 t d^{2}+12 n, \quad \sigma(X)=-8 n
$$

such that the canonical class $K_{X}$ has divisibility $d$. 
Proof. The proof is similar to the proof of Theorem 19. We can write $d=2 k+1$ with $k \geq 0$.

Case: $n=2 m+1$ is odd, where $m \geq 1$. In the proof of Theorem 15, a homotopy elliptic surface $M$ with $\chi_{h}(M)=n$ was constructed from the elliptic surface $E(n)$ by doing knot surgery along a general fibre $F$ with a fibred knot $K_{1}$ of genus $g_{1}=2 \mathrm{~km}+k+1$ and a further knot surgery along a rim torus $R$ with a fibred knot $K_{2}$ of genus $g_{2}=2 k+1=d$. The canonical class is given by

$$
K_{M}=(2 m+1)(2 k+1) F+2(2 k+1) R=(2 m+1) d F+2 d R .
$$

There exists a symplectically embedded 2-sphere $S$ in $E(n)$ of self-intersection -2 that sews together with a Seifert surface for $K_{2}$ to give a symplectic surface $C$ in $M$ of genus $d$ and self-intersection -2 that intersects the rim torus $R$ once. By smoothing the double point, we get a symplectic surface $\Sigma_{M}$ in $M$ of genus $g=d+1$ and self-intersection 0 that represents $C+R$. Using a cusp that intersects $\Sigma_{M}$ once, it follows as above that the complement $M \backslash \Sigma_{M}$ is simply connected.

Let $t \geq 1$ be an arbitrary integer and $K_{3}$ the $(2 h+1,-2)$-torus knot of genus $h=t d$. We consider the generalized fibre sum $X=M \#_{\Sigma_{M}=\Sigma_{S}} Y_{g, h}$, where $g=d+1$. Then $X$ is a simply connected symplectic 4-manifold with invariants

$$
c_{1}^{2}(X)=8 t d^{2}, \quad e(X)=4 t d^{2}+12 n, \quad \sigma(X)=-8 n .
$$

The canonical class is given by

$$
K_{X}=K_{M}+2 t d \Sigma_{M}=d\left((2 m+1) F+2 R+2 t \Sigma_{M}\right) .
$$

Hence $K_{X}$ has divisibility $d$, since the class $(2 m+1) F+2 R+2 t \Sigma_{M}$ twice intersects $\Sigma_{M}$ and has intersection $(2 m+1)$ with a surface coming from a section of $E(n)$ and a Seifert surface for $K_{1}$.

Case: $n=2 m$ is even, where $m \geq 1$. This case can be proved similarly. By doing a logarithmic transform on the fibre $F$ in $E(n)$ and two further knot surgeries with a fibred knot $K_{1}$ of genus $g_{1}=4 k m+k+2$ on the multiple fibre $f$ and with a fibred knot $K_{2}$ of genus $g_{2}=2 k+1=d$ along a rim torus $R$, we get a homotopy elliptic surface $M$ with $\chi_{h}(M)=n$ and canonical class $K_{M}=(4 m+1) d f+2 d R$. The same construction as above yields a simply connected symplectic 4-manifold $X$ with invariants

$$
c_{1}^{2}(X)=8 t d^{2}, \quad e(X)=4 t d^{2}+12 n, \quad \sigma(X)=-8 n .
$$

The canonical class is given by

$$
K_{X}=K_{M}+2 t d \Sigma_{M}=d\left((4 m+1) f+2 R+2 t \Sigma_{M}\right) .
$$

Hence $K_{X}$ again has divisibility $d$. 
Example 23 (nonspin homotopy Horikawa surfaces). The invariants of the manifolds in Theorem 22 are

$$
c_{1}^{2}(X)=8 t d^{2} \quad \text { and } \quad \chi_{h}(X)=t d^{2}+n .
$$

Similarly to Example 20, this implies that for every pair $d, t \geq 1$ of positive integers with $d$ odd and $t$ arbitrary, there exists a nonspin symplectic homotopy Horikawa surface $X$ on the Noether line $c_{1}^{2}=2 \chi_{h}-6$ with invariants

$$
c_{1}^{2}(X)=8 t d^{2} \quad \text { and } \quad \chi_{h}(X)=4 t d^{2}+3,
$$

whose canonical class has divisibility $d$. Note that for every integer $s \geq 1$ there exists a nonspin complex Horikawa surface $M$ [Horikawa 1976a] with invariants

$$
c_{1}^{2}(M)=8 s \quad \text { and } \quad \chi_{h}(M)=4 s+3 .
$$

If $d>1$ and $t$ is an arbitrary integer, we get nonspin homotopy Horikawa surfaces with $s=t d^{2}$ whose canonical classes have divisibility $d$. By the argument from before, these 4-manifolds cannot be diffeomorphic to complex Horikawa surfaces.

With different constructions, it is possible to find examples of simply connected symplectic 4-manifolds with canonical class of odd divisibility, $c_{1}^{2}>0$ and signature not divisible by 8; see [Hamilton 2008, Section VI.2.3]. However, many cases remain uncovered. For example, we could not answer this:

Question 24. For a given odd integer $d>1$, is there a simply connected symplectic 4-manifold $M$ with $c_{1}^{2}(M)=d^{2}$ whose canonical class has divisibility $d$ ?

Note that there is a trivial example for $d=3$, namely $\mathbb{C} P^{2}$.

\section{Construction of inequivalent symplectic structures}

In this section we prove a result similar to [Smith 2000, Theorem 1.5], which can be used to show that certain 4-manifolds $X$ admit inequivalent symplectic structures, where equivalence is defined as follows (see [McMullen and Taubes 1999]).

Definition 25. Two symplectic forms on a closed oriented 4-manifold $M$ are called equivalent if they can be made identical by a combination of deformations through symplectic forms and orientation-preserving self-diffeomorphisms of $M$.

The canonical classes of equivalent symplectic forms have the same (maximal) divisibility as elements of $H^{2}(M ; \mathbb{Z})$. This follows because deformations do not change the canonical class and the application of an orientation preserving selfdiffeomorphism does not change the divisibility.

Lemma 26. Let $(M, \omega)$ be a symplectic 4-manifold with canonical class $K$. Then the symplectic structure $-\omega$ has canonical class $-K$. 
Proof. Let $J$ be an almost complex structure on $M$ compatible with $\omega$. Then $-J$ is an almost complex structure compatible with $-\omega$. The complex vector bundle $(T M,-J)$ is the conjugate bundle to $(T M, J)$. By [Milnor and Stasheff 1974], this implies that $c_{1}(T M,-J)=-c_{1}(T M, J)$. Since the canonical class is minus the first Chern class of the tangent bundle, the claim follows.

Let $M_{K} \times S^{1}$ be a 4-manifold used in knot surgery, where $K$ is a fibred knot of genus $h$. Let $T_{K}$ be a section of the fibre bundle

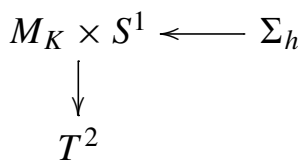

and let $B_{K}$ be a fibre. We fix an orientation on $T_{K}$ and choose the orientation on $B_{K}$ so that $T_{K} \cdot B_{K}=+1$. There exist symplectic structures on $M_{K} \times S^{1}$ such that both the fibre and the section are symplectic. We can choose a symplectic structure $\omega^{+}$that restricts to both $T_{K}$ and $B_{K}$ as a positive volume form with respect to the orientations. It has canonical class $K^{+}=(2 h-2) T_{K}$ by the adjunction formula. We also define the symplectic form $\omega^{-}=-\omega^{+}$. It restricts to a negative volume form on $T_{K}$ and $B_{K}$. The canonical class of this symplectic structure is $K^{-}=-(2 h-2) T_{K}$. Let $X$ be a closed oriented 4-manifold with torsion-free cohomology that contains an embedded oriented torus $T_{X}$ of self-intersection 0 , representing an indivisible homology class. We form the oriented 4-manifold

$$
X_{K}=X \#_{T_{X}=T_{K}}\left(M_{K} \times S^{1}\right),
$$

by doing the generalized fibre sum along the pair $\left(T_{X}, T_{K}\right)$ of oriented tori. Suppose that $X$ has a symplectic structure $\omega_{X}$ such that $T_{X}$ is symplectic. We consider two cases: If $\omega_{X}$ restricts to a positive volume form on $T_{X}$, we can glue $\omega_{X}$ to the symplectic form $\omega^{+}$on $M_{K} \times S^{1}$ to get a symplectic structure $\omega_{X_{K}}^{+}$on $X_{K}$. The canonical class of this symplectic structure is $K_{X_{K}}^{+}=K_{X}+2 h T_{X}$, as seen above; see Equation (3).

Lemma 27. Suppose that $\omega_{X}$ restricts to a negative volume form on $T_{X}$. We can glue $\omega_{X}$ to the symplectic form $\omega^{-}$on $M_{K} \times S^{1}$ to get a symplectic structure $\omega_{X_{K}}^{-}$ on $X_{K}$. The canonical class of $\omega_{X_{K}}^{-}$is $K_{X_{K}}^{-}=K_{X}-2 h T_{X}$.

Proof. We use Lemma 26 twice: The symplectic form $-\omega_{X}$ restricts to a positive volume form on $T_{X}$. We can glue this symplectic form to the symplectic form $\omega^{+}$ on $M_{K} \times S^{1}$, which also restricts to a positive volume form on $T_{K}$. By the standard formula (3), the canonical class of the resulting symplectic form on $X_{K}$ is

$$
K=-K_{X}+2 h T_{X}
$$


The symplectic form $\omega_{X_{K}}^{-}$we want to consider is minus the symplectic form we have just constructed. Hence its canonical class is $K_{X_{K}}^{-}=K_{X}-2 h T_{X}$.

Lemma 28. Suppose $(M, \omega)$ is a closed symplectic 4-manifold with canonical class $K_{M}$. Suppose $M$ contains pairwise disjoint embedded oriented Lagrangian surfaces $T_{1}, \ldots, T_{r+1}$ (with $r \geq 1$ ) such that

- the classes of the surfaces $T_{1}, \ldots, T_{r}$ are linearly independent in $H_{2}(M ; \mathbb{R})$, and

- the surface $T_{r+1}$ is homologous to $a_{1} T_{1}+\cdots+a_{r} T_{r}$, where all coefficients $a_{1}, \ldots, a_{r}$ are positive integers.

Then for every nonempty subset $S \subset\left\{T_{1}, \ldots, T_{r}\right\}$, there exists a symplectic form $\omega_{S}$ on $M$ such that

- all surfaces $T_{1}, \ldots, T_{r+1}$ are symplectic, and

- the symplectic form $\omega_{S}$ induces on the surfaces in $S$ and the surface $T_{r+1}$ a positive volume form and on the remaining surfaces in $\left\{T_{1}, \ldots, T_{r}\right\} \backslash S$ a negative volume form.

Also, the canonical classes of the symplectic structures $\omega_{S}$ are all equal to $K_{M}$. We can also assume that any given closed oriented surface in $M$ that is disjoint from the surfaces $T_{1}, \ldots, T_{r+1}$ and is symplectic with respect to $\omega$ stays symplectic for $\omega_{S}$ with the same sign as the induced volume form.

Proof. The proof is similar to the proof of [Gompf 1995, Lemma 1.6]. We can assume that $S=\left\{T_{s+1}, \ldots, T_{r}\right\}$ with $s+1 \leq r$. Let

$$
c=\sum_{i=1}^{s} a_{i} \quad \text { and } \quad c^{\prime}=\sum_{i=s+1}^{r-1} a_{i} .
$$

Since the classes of the surfaces $T_{1}, \ldots, T_{r}$ are linearly independent in $H_{2}(M ; \mathbb{R})$ and $H_{D R}^{2}(M)$ is the dual space of $H_{2}(M ; \mathbb{R})$, there exists a closed 2-form $\eta$ on $M$ such that

$$
\int_{T_{i}} \eta= \begin{cases}-1 & \text { for } i=1, \ldots, s, \\ +1 & \text { for } i=s+1, \ldots, r-1, \\ c+1 & \text { for } i=r \\ c^{\prime}+1 & \text { for } i=r+1,\end{cases}
$$

Note that we can choose the value of $\eta$ on $T_{1}, \ldots, T_{r}$ arbitrarily. The value on $T_{r+1}$ is then determined by $T_{r+1}=a_{1} T_{1}+\cdots+a_{r} T_{r}$. We can choose symplectic forms $\omega_{i}$ on each $T_{i}$ such that

$$
\int_{T_{i}} \omega_{i}=\int_{T_{i}} \eta \quad \text { for all } i=1, \ldots, r+1 .
$$


The symplectic form $\omega_{i}$ induces on $T_{i}$ a negative volume form if $i \leq s$ and a positive volume form if $i \geq s+1$. The difference $\omega_{i}-j_{i}^{*} \eta$, where $j_{i}: T_{i} \rightarrow M$ is the embedding, has vanishing integral and hence is an exact 2-form on $T_{i}$ of the form $d \alpha_{i}$. We can extend each $\alpha_{i}$ to a small tubular neighbourhood of $T_{i}$ in $M$, cut it off differentiably in a slightly larger tubular neighbourhood and extend by 0 to all of $M$. We can do this such that the tubular neighbourhoods of $T_{1}, \ldots, T_{r+1}$ are pairwise disjoint. Define the closed 2-form $\eta^{\prime}=\eta+\sum_{i=1}^{r+1} d \alpha_{i}$ on $M$. Then

$$
j_{i}^{*} \eta^{\prime}=j_{i}^{*} \eta+d \alpha_{i}=\omega_{i} .
$$

The closed 2-form $\omega^{\prime}=\omega+t \eta^{\prime}$ is symplectic for small values of $t$. Since the surfaces $T_{i}$ are Lagrangian, we have $j_{i}^{*} \omega=0$ and hence $j_{i}^{*} \omega^{\prime}=t \omega_{i}$. This implies that $\omega^{\prime}$ is for small values $t>0$ a symplectic form on $M$ that induces a volume form on $T_{i}$ of the same sign as $\omega_{i}$ for all $i=1, \ldots, r+1$. The claim about the canonical class follows because the symplectic structures $\omega_{S}$ are constructed by a deformation of $\omega$. We can also choose $t>0$ small enough so that $\omega^{\prime}$ still restricts to a symplectic form on any given symplectic surface disjoint from the tori without changing the sign of the induced volume form on this surface.

This construction will be used as follows: Suppose that $\left(V_{1}, \omega_{1}\right)$ and $\left(V_{2}, \omega_{2}\right)$ are symplectic 4-manifolds such that $V_{1}$ contains an embedded Lagrangian torus $T_{1}$ and $V_{2}$ contains an embedded symplectic torus $T_{2}$, both oriented and of selfintersection 0 . Let $W$ denote the smooth oriented 4-manifold $V_{1} \#_{T_{1}=T_{2}} V_{2}$ obtained as a generalized fibre sum. By Lemma 28, there exist small perturbations of $\omega_{1}$ to new symplectic forms $\omega_{1}^{+}$and $\omega_{1}^{-}$on the manifold $V_{1}$ such that the torus $T_{1}$ becomes symplectic with positive and negative induced volume form, respectively. By the Gompf construction, it is then possible to define two symplectic forms on the same oriented 4-manifold $W$ :

- The symplectic forms $\omega_{1}^{+}$and $\omega_{2}$ determine a symplectic form on $W$.

- The symplectic forms $\omega_{1}^{-}$and $-\omega_{2}$ determine a symplectic form on $W$.

Hence the symplectic forms on the first manifold differ only by a small perturbation, while on the second manifold they differ by the sign. Similarly, the canonical classes of both perturbed symplectic forms on $V_{1}$ are the same, while they differ by the sign on $V_{2}$. If additional tori exist and suitable fibre sums are performed, it is possible to end up with two or more inequivalent symplectic forms on the same 4-manifold, distinguished by the divisibilities of their canonical classes.

To define the configuration of tori we want to consider, recall that the nucleus $N(n)$ is the smooth manifold with boundary defined as a regular neighbourhood of a cusp fibre and a section in the simply connected elliptic surface $E(n)$; see [Gompf 1991]. It contains an embedded torus given by a regular fibre homologous to the cusp. It also contains two embedded disks of self-intersection -1 that bound 
vanishing cycles on the torus. The vanishing cycles are the simple closed loops given by the factors in $T^{2}=S^{1} \times S^{1}$.

Definition 29 (Lagrangian triple). Let $(M, \omega)$ be a symplectic 4-manifold. Given an integer $a \geq 1$, a Lagrangian triple consists of three pairwise disjoint oriented Lagrangian tori $T_{1}, T_{2}$ and $R$ embedded in $M$ with the following properties:

- All three tori have self-intersection zero and represent indivisible classes in integral homology.

- $T_{1}$ and $T_{2}$ are linearly independent over $\mathbb{Q}$ and $R$ is homologous to $a T_{1}+T_{2}$.

- There exists an embedded nucleus $N(2) \subset M$ that contains $R$, corresponding to a general fibre. Let $S$ denote the 2-sphere in $N(2)$ of self-intersection -2 , corresponding to a section. In addition to intersecting $R$, this sphere intersects $T_{2}$ transversely once. The torus $T_{2}$ is disjoint from the vanishing disks of $R$, coming from the cusp in $N(2)$.

- The torus $T_{1}$ is disjoint from the nucleus $N(2)$ above, and there exists an embedded 2-sphere $S_{1}$ in $M$, also disjoint from $N(2)$, that intersects $T_{1}$ transversely and positively once.

See Figure 1. The assumptions imply that $S_{1} T_{2}=S_{1}\left(R-a T_{1}\right)=-a$.

Example 30. Let $M$ be the elliptic surface $E(n)$ with $n \geq 2$. In this example we show that $E(n)$ contains $n-1$ disjoint Lagrangian triples $\left(T_{1}^{i}, T_{2}^{i}, R^{i}\right)$ as above, where $R^{i}$ is homologous to $a_{i} T_{1}^{i}+T_{2}^{i}$ for $i=1, \ldots, n-1$. The integers $a_{i}>0$ can be chosen arbitrarily and for each triple independently. In this case both $T_{1}^{i}$ and $R^{i}$ are contained in disjoint embedded nuclei $N(2)$. Together with their dual 2-spheres they realize $2(n-1) H$-summands in the intersection form of $E(n)$. In

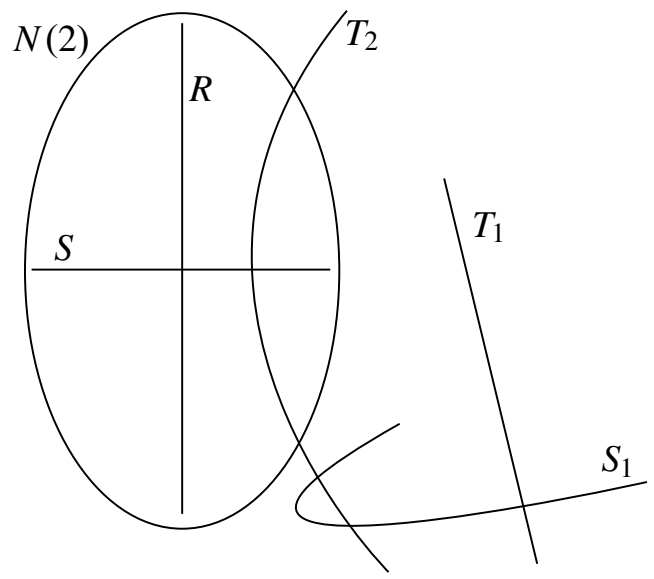

Figure 1. Lagrangian triple. 
particular, the tori in different triples are linearly independent. We can also ensure that all Lagrangian tori and the 2-spheres that intersect them once are disjoint from the nucleus $N(n) \subset E(n)$, defined as a regular neighbourhood of a cusp fibre and a section in $E(n)$.

The construction is similar to [Gompf and Mrowka 1993, Section 2] and is done by induction. Suppose the Lagrangian triples are already constructed for $E(n)$ and consider a splitting of $E(n+1)$ as a fibre sum $E(n+1)=E(n) \#_{F=F} E(1)$ along general fibres $F$. We choose fibred tubular neighbourhoods for the general fibres in $E(n)$ and $E(1)$. The boundary of $E(1) \backslash \operatorname{int} v F$ is diffeomorphic to $F \times S^{1}$. Let $\gamma_{1}$ and $\gamma_{2}$ be two simple closed loops spanning the torus $F$, and let $m$ be the meridian to $F$ that spans the remaining $S^{1}$ factor. We consider the three tori

$$
V_{0}=\gamma_{1} \times \gamma_{2}, \quad V_{1}=\gamma_{1} \times m, \quad V_{2}=\gamma_{2} \times m .
$$

The tori are made disjoint by pushing them inside a collar of the boundary into the interior of $E(1) \backslash$ int $v F$ such that $V_{2}$ is the innermost and $V_{0}$ the outermost (closest to the boundary). The torus $V_{0}$ can be assumed symplectic, while $V_{1}$ and $V_{2}$ are rim tori that can be assumed Lagrangian. Similarly the boundary of $E(n) \backslash$ int $v F$ is diffeomorphic to $F \times S^{1}$, where $F$ is spanned by the circles $\gamma_{1}$ and $\gamma_{2}$ and $S^{1}$ by the circle $m$ and corresponding circles get identified in the gluing of the fibre sum. In the interior of $E(n) \backslash$ int $\nu F$ we consider three tori $V_{0}, V_{1}, V_{2}$ as above which get identified with the corresponding tori on the $E(1)$ side in the gluing. On the $E(n)$ side, the torus $V_{0}$ is the innermost and $V_{2}$ the outermost.

We can choose elliptic fibrations such that near the general fibre $F$ there exist two cusp fibres in $E(1)$ and three cusp fibres in $E(n)$. This is possible because $E(m)$ has an elliptic fibration with $6 m$ cusp fibres for all $m$; see [Gompf and Stipsicz 1999, Corollary 7.3.23]. The corresponding vanishing disks can be assumed pairwise disjoint. We can also choose three disjoint sections for the elliptic fibration on $E(1)$ and one section for $E(n)$.

The nuclei can now be defined as follows: The nucleus $N(n+1)$ containing $V_{0}$ has a dual $-(n+1)$-sphere sewed together from sections on each side of the fibre sum. The vanishing disks for $V_{0}$ come from the first cusp in $E(n)$. The nucleus $N(2)$ containing $V_{1}$ has a dual -2 -sphere sewed together from two vanishing cycles parallel to $\gamma_{2}$ coming from the first cusp in $E(1)$ and the second cusp in $E(n)$. The vanishing disks for $V_{1}$ come from the second section of $E(1)$ and from the vanishing cycle parallel to $\gamma_{1}$ of the second cusp in $E(n)$. The nucleus $N(2)$ containing $V_{2}$ has a dual -2 -sphere sewed together from two vanishing cycles parallel to $\gamma_{1}$ coming from the second cusp in $E(1)$ and the third cusp in $E(n)$. The vanishing disks for $V_{2}$ come from the third section of $E(1)$ and from the vanishing cycle parallel to $\gamma_{2}$ of the second cusp in $E(1)$. 
To define the Lagrangian triple $\left(T_{1}, T_{2}, R\right)$, let $T_{1}=V_{1}$ and $R=V_{2}$. Denote by $c_{a}: S^{1} \rightarrow F=S^{1} \times S^{1}$ the embedded curve given by the $(-a, 1)$-torus knot, and let $T_{2}$ denote the Lagrangian rim torus $T_{2}=c_{a} \times m$ in the collar above. Then $T_{2}$ represents the class $-a T_{1}+R$; hence $R=a T_{1}+T_{2}$. The torus $T_{2}$ has one positive transverse intersection with the sphere in the nucleus containing $R$ and $a$ negative transverse intersections with the sphere in the nucleus containing $T_{1}$.

Remark 31. To find more general examples of symplectic 4-manifolds containing Lagrangian triples, suppose that $Y$ is an arbitrary closed symplectic 4-manifold that contains an embedded symplectic torus $T_{Y}$ of self-intersection 0 , representing an indivisible class. Then the symplectic generalized fibre sum $Y \#_{T_{Y}=F} E(n)$ also contains $n-1$ Lagrangian triples.

Suppose $(M, \omega)$ is a simply connected symplectic 4-manifold that contains a Lagrangian triple $T_{1}, T_{2}, R$. Let $K_{1}$ and $K_{2}$ be fibred knots of genera $h_{1}$ and $h_{2}$ to be chosen later. Consider the associated oriented 4-manifolds $M_{K_{i}} \times S^{1}$ as in the knot surgery construction, and denote sections of the fibre bundles

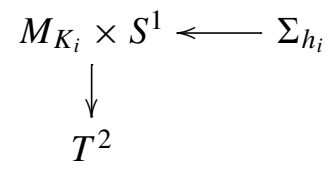

by $T_{K_{i}}$, which are tori of self-intersection 0 . We choose an orientation on each torus $T_{K_{i}}$. Note that the Lagrangian tori $T_{1}$ and $T_{2}$ in $M$ are oriented a priori.

We construct a smooth oriented 4-manifold $X$ in three steps as follows: For an integer $m \geq 1$, consider the elliptic surface $E(m)$ and denote an oriented general fibre by $F$. Let $M_{0}$ denote the smooth generalized fibre sum $M_{0}=E(m) \#_{F=R} M$. The gluing diffeomorphism is chosen as follows: The push-offs $R^{\prime}$ and $F^{\prime}$ into the boundary of the tubular neighbourhoods $v R$ and $v F$ each contain a pair of vanishing cycles. We choose the gluing so that the push-offs and the vanishing cycles get identified. The corresponding vanishing disks then sew together pairwise to give two embedded spheres of self-intersection -2 in $M_{0}$, which can be assumed disjoint by choosing two different push-offs given by the same trivializations.

Denote the torus in $M_{0}$ coming from the push-off $R^{\prime}$ by $R_{0}$. Consider the tori $T_{1}$ and $T_{2}$ in $M_{0}$. Then $R_{0}$ is still homologous to $a T_{1}+T_{2}$ in $M_{0}$, because the difference could only be a rim torus by [Hamilton 2008, Section V.3], which must have nonzero intersection with one of the two vanishing spheres in $M_{0}$. This is excluded by our assumptions on Lagrangian triples. In the second step of the construction, we do a knot surgery with the fibred knot $K_{1}$ along the torus $T_{1}$ in $M_{0}$ to get the oriented 4-manifold $M_{1}=M_{0} \#_{T_{1}=T_{K_{1}}}\left(M_{K_{1}} \times S^{1}\right)$. The manifold $M_{1}$ contains a torus, which we still denote by $T_{2}$. We do a knot surgery with the fibred 
knot $K_{2}$ along the torus $T_{2}$ to get the oriented 4-manifold $X=M_{1} \#_{T_{2}=T_{K_{2}}}\left(M_{K_{2}} \times\right.$ $\left.S^{1}\right)$.

\section{Lemma 32. The closed oriented 4-manifold}

$$
X=E(m) \#_{F=R} M \#_{T_{1}=T_{K_{1}}}\left(M_{K_{1}} \times S^{1}\right) \#_{T_{2}=T_{K_{2}}}\left(M_{K_{2}} \times S^{1}\right)
$$

is simply connected.

Proof. The existence of the sphere $S$ shows that $M \backslash R$ is simply connected. Since $E(m) \backslash F$ is simply connected, it follows that $M_{0}$ is simply connected.

The sphere $S$ and a section for the elliptic fibration on $E(m)$ sew together to give an embedded sphere $S_{2}$ in $M_{0}$ of self-intersection $-(m+2)$. The sphere $S_{1}$ in $M$ is disjoint from $R$ and hence is still contained in $M_{0}$. These spheres have the following intersections:

- The sphere $S_{1}$ intersects $T_{1}$ transversely once, has intersection $-a$ with $T_{2}$, and is disjoint from $R_{0}$.

- The sphere $S_{2}$ intersects $R_{0}$ and $T_{2}$ transversely once and is disjoint from $T_{1}$.

The sphere $S_{1}$ shows that $M_{0} \backslash T_{1}$ is simply connected and hence $M_{1}$ is simply connected. The sphere $S_{2}$ in $M_{0}$ is disjoint from $T_{1}$ and hence is still contained in $M_{1}$ and intersects $T_{2}$ once. By the same argument, this shows that the manifold $X$ is simply connected.

We define two symplectic forms $\omega_{X}^{+}$and $\omega_{X}^{-}$on $X$ : By Lemma 28 there exist two symplectic structures $\omega_{+}$and $\omega_{-}$on $M$ with the same canonical class $K_{M}$ as $\omega$ such that

- the tori $T_{1}, T_{2}$ and $R$ are symplectic with respect to both symplectic forms,

- the form $\omega_{+}$induces on $T_{1}, T_{2}$ and $R$ a positive volume form, and

- the form $\omega_{-}$induces on $T_{1}$ a negative volume form and on $T_{2}$ and $R$ a positive volume form.

We can also choose the sphere $S$ to be symplectic with positive volume form in both cases.

On the elliptic surface $E(m)$, we can choose a symplectic (Kähler) form $\omega_{E}$ that restricts to a positive volume form on the oriented fibre $F$. It has canonical class $K_{E}=(m-2) F$. We can glue both symplectic forms $\omega_{+}$and $\omega_{-}$on $M$ to the symplectic form $\omega_{E}$ on $E(m)$ to get symplectic forms $\omega_{0}^{+}$and $\omega_{0}^{-}$on the 4manifold $M_{0}$. The canonical class for both symplectic forms on $M_{0}$ is given by $K_{M_{0}}=K_{M}+m R_{0}$; see [Fintushel and Stern 2001, proof of Lemma 2.2]. Since rim tori exist in this fibre sum, Theorem 11 cannot be applied directly. However, the formula remains correct because rim tori do not contribute in this case; for details see [Hamilton 2008, Section V.6.1]. 
We want to extend the symplectic forms to the 4-manifold $X$ : We choose in each fibre bundle $M_{K_{i}} \times S^{1}$ a fibre $B_{K_{i}}$ and orient the surface $B_{K_{i}}$ so that $T_{K_{i}} \cdot B_{K_{i}}=+1$ with the chosen orientation on $T_{K_{i}}$. There exist symplectic structures on the closed 4-manifolds $M_{K_{i}} \times S^{1}$ such that both the section and the fibre are symplectic. On $M_{K_{1}} \times S^{1}$, we choose two symplectic forms $\omega_{1}^{ \pm}$: The form $\omega_{1}^{+}$induces a positive volume form on both $T_{K_{1}}$ and $B_{K_{1}}$. It has canonical class $K_{1}^{+}=\left(2 h_{1}-2\right) T_{K_{1}}$. The form $\omega_{1}^{-}$is given by $-\omega_{1}^{+}$. It induces a negative volume form on both $T_{K_{1}}$ and $B_{K_{1}}$ and has canonical class $K_{1}^{-}=-\left(2 h_{1}-2\right) T_{K_{1}}$.

On the manifold $M_{K_{2}} \times S^{1}$ we only choose a symplectic form $\omega_{2}$ that induces a positive volume form on $T_{K_{2}}$ and $B_{K_{2}}$. The canonical class is $K_{2}=\left(2 h_{2}-2\right) T_{K_{2}}$.

The oriented torus $T_{1}$ in $M_{0}$ is symplectic for both forms $\omega_{0}^{ \pm}$constructed as above so that $\omega_{0}^{+}$induces a positive volume form and $\omega_{0}^{-}$a negative volume form. Gluing $\omega_{0}^{+}$to $\omega_{1}^{+}$and $\omega_{0}^{-}$to $\omega_{1}^{-}$, Lemma 27 implies that the closed oriented 4-manifold $M_{1}$ has two symplectic structures with canonical classes

$$
K_{M_{1}}^{+}=K_{M}+m R_{0}+2 h_{1} T_{1} \quad \text { and } \quad K_{M_{1}}^{-}=K_{M}+m R_{0}-2 h_{1} T_{1} .
$$

The torus $T_{2}$ can be considered as a symplectic torus in $M_{1}$ such that both symplectic structures induce positive volume forms, since we can assume that the symplectic forms on $M_{1}$ are still of the form $\omega_{0}^{+}$and $\omega_{0}^{-}$in a neighbourhood of $T_{2}$. Hence on the generalized fibre sum $X=M_{1} \#_{T_{2}=T_{K_{2}}} M_{K_{2}} \times S^{1}$, we can glue each of the two symplectic forms on $M_{1}$ to the symplectic form $\omega_{2}$ on $M_{K_{2}} \times S^{1}$. We get two symplectic structures on $X$ with canonical classes

$$
\begin{aligned}
& K_{X}^{+}=K_{M}+m R_{0}+2 h_{1} T_{1}+2 h_{2} T_{2}, \\
& K_{X}^{-}=K_{M}+m R_{0}-2 h_{1} T_{1}+2 h_{2} T_{2} .
\end{aligned}
$$

This can be written using $R_{0}=a T_{1}+T_{2}$ as

$$
\begin{aligned}
& K_{X}^{+}=K_{M}+\left(2 h_{1}+a m\right) T_{1}+\left(2 h_{2}+m\right) T_{2}, \\
& K_{X}^{-}=K_{M}+\left(-2 h_{1}+a m\right) T_{1}+\left(2 h_{2}+m\right) T_{2} .
\end{aligned}
$$

Theorem 33. Suppose $(M, \omega)$ is a simply connected symplectic 4-manifold that contains a Lagrangian triple $T_{1}, T_{2}, R$ such that $R$ is homologous to a $T_{1}+T_{2}$. Let $m$ be a positive integer, and let $K_{1}$ and $K_{2}$ be fibred knots of genus $h_{1}$ and $h_{2}$. Then the closed oriented 4-manifold

$$
X=E(m) \#_{F=R} M \#_{T_{1}=T_{K_{1}}}\left(M_{K_{1}} \times S^{1}\right) \#_{T_{2}=T_{K_{2}}}\left(M_{K_{2}} \times S^{1}\right)
$$

is simply connected and admits symplectic structures $\omega_{X}^{+}$and $\omega_{X}^{-}$with canonical classes

$$
\begin{aligned}
& K_{X}^{+}=K_{M}+\left(2 h_{1}+a m\right) T_{1}+\left(2 h_{2}+m\right) T_{2}, \\
& K_{X}^{-}=K_{M}+\left(-2 h_{1}+a m\right) T_{1}+\left(2 h_{2}+m\right) T_{2} .
\end{aligned}
$$


Remark 34. Instead of doing the generalized fibre sum with $E(m)$ in the first step of the construction, we could also do a knot surgery with a fibred knot $K_{0}$ of genus $h_{0} \geq 1$. This has the advantage that both $c_{1}^{2}$ and the signature do not change under the construction. However, the sphere $S_{2}$ in $M_{0}$ is then replaced by a surface of genus $h_{0}$ sewed together from the sphere $S$ in $M$ and a Seifert surface for $K_{0}$. Hence it is no longer clear that $M_{1} \backslash T_{2}$ and $X$ are simply connected.

The following two surfaces are useful for determining the divisibility of the canonical classes in Theorem 33.

Lemma 35. There is an oriented surface $C_{2}$ in $X$ that has intersection $C_{2} T_{2}=1$ and is disjoint from $T_{1}$.

The surface $C_{2}$ is sewed together from the sphere $S_{2}$ and a Seifert surface for $K_{2}$.

Lemma 36. There is an oriented surface $C_{1}$ in $X$ that has intersection $C_{1} T_{1}=1$ and is disjoint from $T_{2}$.

Proof. The surface $C_{1}$ can be constructed explicitly as follows: In the nucleus $N(2) \subset M$ containing $R$, we can find a surface of some genus homologous to $a S$ and intersecting both $R$ and $T_{2}$ in $a$ positive transverse intersections. Tubing this surface to the sphere $S_{1}$, we get a surface $A$ in $M$ that has intersection number $A T_{2}=0$ and intersects $T_{1}$ transversely once. By increasing the genus we can make $A$ disjoint from $T_{2}$. The surface $A$ still intersects the torus $R$ at $a$ points. Sewing the surface $A$ to a surface in $E(m)$ homologous to $a$ times a section, we get a surface $B$ in $M_{0}$ disjoint from $T_{2}$ and intersecting $T_{1}$ once. Sewing this surface to a Seifert surface for $K_{1}$ we get a surface $C_{1}$ in $X$ with $C_{1} T_{1}=1$ disjoint from $T_{2}$.

\section{Examples of inequivalent symplectic structures}

Definition 37 (the set $Q$ ). Let $N \geq 0$ and $d \geq 1$ be integers, and let $d_{0}, \ldots, d_{N}$ be positive integers dividing $d$, where $d=d_{0}$. If $d$ is even, assume that all $d_{1}, \ldots, d_{N}$ are even. We define a set $Q$ of positive integers as follows:

- If $d$ is either odd or not divisible by 4 , let $Q$ be the set consisting of the greatest common divisors of all (nonempty) subsets of $\left\{d_{0}, \ldots, d_{N}\right\}$.

- If $d$ is divisible by 4 , we can assume by reordering that $d_{1}, \ldots, d_{s}$ are those elements such that $d_{i}$ is divisible by 4 , while $d_{s+1}, \ldots, d_{N}$ are those elements such that $d_{i}$ is not divisible by 4 , where $s \geq 0$ is some integer. Then $Q$ is defined as the set of integers consisting of the greatest common divisors of all (nonempty) subsets of $\left\{d_{0}, \ldots, d_{s}, 2 d_{s+1}, \ldots, 2 d_{N}\right\}$.

We can now state the main theorem on the existence of inequivalent symplectic structures on homotopy elliptic surfaces. 
Theorem 38. Let $N$ and $d \geq 1$ be integers, and let $d_{0}, \ldots, d_{N}$ be positive integers dividing $d$ as in Definition 37. Let $Q$ be the associated set of greatest common divisors. Choose an integer $n \geq 3$ as follows:

- If $d$ is odd, let $n$ be an arbitrary integer with $n \geq 2 N+1$.

- If $d$ is even, let $n$ be an even integer with $n \geq 3 N+1$.

Then there exists a homotopy elliptic surface $W$ with $\chi_{h}(W)=n$ and the property that for each integer $q \in Q$, the manifold $W$ admits a symplectic structure whose canonical class $K$ has divisibility equal to $q$. Hence $W$ admits at least $|Q|$ inequivalent symplectic structures.

Proof. The proof splits into three cases depending on the parity of $d$. In each case we follow the construction in Section 8, starting from the manifold $M=E(l)$, where $l$ is an integer no less than $N+1$. By Example 30, $E(l)$ contains $N$ pairwise disjoint Lagrangian triples $T_{1}^{i}, T_{2}^{i}, R^{i}$, where $R^{i}$ is homologous to $a_{i} T_{1}^{i}+T_{2}^{i}$ for indices $i=1, \ldots, N$. The construction is done on each triple separately ${ }^{2}$ and involves knot surgeries along $T_{1}^{i}$ and $T_{2}^{i}$ with fibred knots of respective genus $h_{i}$ and $h$, as well as fibre summing with elliptic surfaces $E(m)$ along the tori $R^{i}$. The numbers $a_{i}, h_{i}, h$ and $m$ will be fixed in each case.

Case: $d$ is odd. Then all divisors $d_{1}, \ldots, d_{N}$ are odd. Consider the integers

$$
\begin{array}{ll}
m=1, & h=\frac{1}{2}(d-1), \\
a_{i}=d+d_{i}, & h_{i}=\frac{1}{2}\left(d-d_{i}\right) \quad \text { for } 1 \leq i \leq N .
\end{array}
$$

Let $l$ be an integer no less than $N+1$ and do the construction above, starting from the elliptic surface $E(l)$. We get a (simply connected) homotopy elliptic surface $X$ with $\chi_{h}(X)=l+N$. By Theorem 33 the 4-manifold $X$ has $2^{N}$ symplectic structures with canonical classes

$$
\begin{aligned}
K_{X} & =(l-2) F+\sum_{i=1}^{N}\left(\left( \pm 2 h_{i}+a_{i}\right) T_{1}^{i}+(2 h+1) T_{2}^{i}\right) \\
& =(l-2) F+\sum_{i=1}^{N}\left(\left( \pm\left(d-d_{i}\right)+d+d_{i}\right) T_{1}^{i}+d T_{2}^{i}\right) .
\end{aligned}
$$

Here $F$ denotes the torus in $X$ coming from a general fibre in $E(l)$ and the \pm -signs in each summand can be varied independently. We can assume that $F$ is symplectic with positive induced volume form for all $2^{N}$ symplectic structures on $X$. Consider the even integer $l(d-1)+2$, and let $K$ be a fibred knot of genus $g=\frac{1}{2}(l(d-1)+2)$. We do knot surgery with $K$ along the symplectic torus $F$ to get a homotopy elliptic

\footnotetext{
${ }^{2}$ This is only a small generalization of Lemma 28 , because the construction in the proof of this lemma changes the symplectic structure only in a small neighbourhood of the Lagrangian surfaces.
} 
surface $W$ with $\chi_{h}(W)=l+N$ having $2^{N}$ symplectic structures whose canonical classes are given by

$$
\begin{aligned}
K_{W} & =(l-2+2 g) F+\sum_{i=1}^{N}\left(\left( \pm\left(d-d_{i}\right)+d+d_{i}\right) T_{1}^{i}+d T_{2}^{i}\right) \\
& =d l F+\sum_{i=1}^{N}\left(\left( \pm\left(d-d_{i}\right)+d+d_{i}\right) T_{1}^{i}+d T_{2}^{i}\right) .
\end{aligned}
$$

Suppose that $q \in Q$ is the greatest common divisor of certain elements $\left\{d_{i}\right\}_{i \in I}$, where $I$ is a nonempty subset of $\{0, \ldots, N\}$. Let $J$ be the complement of $I$ in $\{0, \ldots, N\}$. Choosing the minus sign for each $i$ in $I$ and the plus sign for each $i$ in $J$ defines a symplectic structure $\omega_{I}$ on $W$ with canonical class given by

$$
K_{W}=d l F+\sum_{i \in I}\left(2 d_{i} T_{1}^{i}+d T_{2}^{i}\right)+\sum_{j \in J}\left(2 d T_{1}^{j}+d T_{2}^{j}\right) .
$$

We claim that the divisibility of $K_{W}$ is equal to $q$. Since $q$ divides $d$ and all integers $d_{i}$ for $i \in I$, the class $K_{W}$ is divisible by $q$. Considering separately the surfaces from Lemmas 35 and 36 for each Lagrangian triple implies that every number that divides $K_{W}$ is odd (since it divides $d$ ) and a common divisor of all $d_{i}$ with indices $i \in I$. This proves the claim in this case.

Case: $d$ is even but not divisible by 4 . We can write $d=2 k$ and $d_{i}=2 k_{i}$ for all $i=1, \ldots, N$. The assumption implies that all integers $k, k_{i}$ are odd. Consider the integers defined by

$$
\begin{aligned}
m & =2, & h & =k-1, \\
a_{i} & =\frac{1}{2}\left(k+k_{i}\right), & h_{i} & =\frac{1}{2}\left(k-k_{i}\right) .
\end{aligned}
$$

Let $l$ be an even integer no less than $N+1$ and consider the construction above, starting from $E(l)$. We get a homotopy elliptic surface $X$ with $\chi_{h}(X)=l+2 N$. The 4-manifold $X$ has $2^{N}$ symplectic structures with canonical classes

$$
\begin{aligned}
K_{X} & =(l-2) F+\sum_{i=1}^{N}\left(\left( \pm 2 h_{i}+2 a_{i}\right) T_{1}^{i}+(2 h+2) T_{2}^{i}\right) \\
& =(l-2) F+\sum_{i=1}^{N}\left(\left( \pm\left(k-k_{i}\right)+k+k_{i}\right) T_{1}^{i}+d T_{2}^{i}\right) .
\end{aligned}
$$

Consider a fibred knot $K$ of genus $g=\frac{1}{2}(l(d-1)+2)$, noting that $l$ is even. Doing knot surgery with $K$ along the symplectic torus $F$ in $X$, we get a homotopy elliptic surface $W$ with $\chi_{h}(W)=l+2 N$ having $2^{N}$ symplectic structures whose canonical 
classes are

$$
\begin{aligned}
K_{W} & =(l-2+2 g) F+\sum_{i=1}^{N}\left(\left( \pm\left(k-k_{i}\right)+k+k_{i}\right) T_{1}^{i}+d T_{2}^{i}\right) \\
& =d l F+\sum_{i=1}^{N}\left(\left( \pm\left(k-k_{i}\right)+k+k_{i}\right) T_{1}^{i}+d T_{2}^{i}\right) .
\end{aligned}
$$

Let $q \in Q$ be the greatest common divisor of elements $d_{i}$, where $i \in I$ for some nonempty index set $I$ with complement $J$ in $\{0, \ldots, N\}$. Choosing the plus and minus signs as before, we get a symplectic structure $\omega_{I}$ on $W$ with canonical class

$$
K_{W}=d l F+\sum_{i \in I}\left(d_{i} T_{1}^{i}+d T_{2}^{i}\right)+\sum_{j \in J}\left(d T_{1}^{i}+d T_{2}^{i}\right)
$$

As above, it follows that the canonical class of $\omega_{I}$ has divisibility equal to $q$.

Case: $d$ is divisible by 4 . We can write $d=2 k$ and $d_{i}=2 k_{i}$ for all $i=1, \ldots, N$. We can assume that the divisors are ordered as in Definition 37, that is, $d_{1}, \ldots, d_{s}$ are those elements such that $d_{i}$ is divisible by 4 while $d_{s+1}, \ldots, d_{N}$ are those elements such that $d_{i}$ is not divisible by 4 . This is equivalent to $k_{1}, \ldots, k_{s}$ being even and $k_{s+1}, \ldots, k_{N}$ odd. Consider the integers defined by

$$
\begin{array}{lll}
a_{i}=\frac{1}{2}\left(k+k_{i}\right) & \text { and } \quad h_{i}=\frac{1}{2}\left(k-k_{i}\right) & \text { for } i=1, \ldots, s, \\
a_{i}=\frac{1}{2}\left(k+2 k_{i}\right) & \text { and } \quad h_{i}=\frac{1}{2}\left(k-2 k_{i}\right) & \text { for } i=s+1, \ldots, N .
\end{array}
$$

We also define $m=2$ and $h=k-1$. Let $l$ be an even integer $\geq N+1$. We consider the same construction as above starting from $E(l)$ to get a homotopy elliptic surface $X$ with $\chi_{h}(X)=l+2 N$ that has $2^{N}$ symplectic structures with canonical classes given by the formula

$$
\begin{gathered}
K_{X}=(l-2) F+\sum_{i=1}^{N}\left(\left( \pm 2 h_{i}+2 a_{i}\right) T_{1}^{i}+(2 h+2) T_{2}^{i}\right) \\
=(l-2) F+\sum_{i=1}^{s}\left(\left( \pm\left(k-k_{i}\right)+k+k_{i}\right) T_{1}^{i}+d T_{2}^{i}\right) \\
+\sum_{i=s+1}^{N}\left(\left( \pm\left(k-2 k_{i}\right)+k+2 k_{i}\right) T_{1}^{i}+d T_{2}^{i}\right) .
\end{gathered}
$$

We then do knot surgery with a fibred knot $K$ of genus $g=\frac{1}{2}(l(d-1)+2)$ along the symplectic torus $F$ in $X$ to get a homotopy elliptic surface $W$ with $\chi_{h}(W)=l+2 N$ 
having $2^{N}$ symplectic structures whose canonical classes are

$$
\begin{gathered}
K_{W}=(l-2+2 g) F+\sum_{i=1}^{N}\left(\left( \pm\left(k-k_{i}\right)+k+k_{i}\right) T_{1}^{i}+d T_{2}^{i}\right) \\
=d l F+\sum_{i=1}^{s}\left(\left( \pm\left(k-k_{i}\right)+k+k_{i}\right) T_{1}^{i}+d T_{2}^{i}\right) \\
+\sum_{i=s+1}^{N}\left(\left( \pm\left(k-2 k_{i}\right)+k+2 k_{i}\right) T_{1}^{i}+d T_{2}^{i}\right) .
\end{gathered}
$$

Let $q$ be an element in $Q$. Note that this time

$$
\begin{array}{rlll}
\left(k-k_{i}\right)+\left(k_{i}+k\right)=d & \text { and } & -\left(k-k_{i}\right)+\left(k_{i}+k\right)=d_{i} & \text { for } i \leq s, \\
\left(k-2 k_{i}\right)+\left(k+2 k_{i}\right)=d & \text { and } & -\left(k-2 k_{i}\right)+\left(k+2 k_{i}\right)=2 d_{i} & \text { for } i \geq s+1 .
\end{array}
$$

Since $q$ is the greatest common divisor of certain elements $d_{i}$ for $i \leq s$ and $2 d_{i}$ for $i \geq s+1$, it follows as above that we can choose the plus and minus signs appropriately to get a symplectic structure $\omega_{I}$ on $W$ whose canonical class has divisibility equal to $q$.

Example 39. Suppose $d=45$ and choose $d_{0}=45, d_{1}=15, d_{2}=9, d_{3}=5$. Then $Q=\{45,15,9,5,3,1\}$, and for every integer $n \geq 7$ there exists a homotopy elliptic surfaces $W$ with $\chi_{h}(W)=n$ that admits at least 6 inequivalent symplectic structures whose canonical classes have divisibilities given by the elements in $Q$. One can also find an infinite family of homeomorphic but nondiffeomorphic manifolds of this kind.

Corollary 40. Let $m \geq 1$ be an arbitrary integer.

- There exist simply connected nonspin 4-manifolds $W$ homeomorphic to the elliptic surfaces $E(2 m+1)$ and $E(2 m+2)_{2}$ that admit at least $2^{m}$ inequivalent symplectic structures.

- There exist simply connected spin 4-manifolds W homeomorphic to E $(6 m-2)$ and $E(6 m)$ that admit at least $2^{2 m-1}$ inequivalent symplectic structures, and there are spin manifolds homeomorphic to $E(6 m+2)$ that admit at least $2^{2 m}$ inequivalent symplectic structures.

Proof. Choose $N$ pairwise different odd prime numbers $p_{1}, \ldots, p_{N}$. Let $d=d_{0}=$ $p_{1} \cdots p_{N}$, and consider the integers $d_{i}$ obtained for $i=1, \ldots, N$ by dividing $d$ by the prime $p_{i}$. Then the associated set $Q$ of greatest common divisors consists of all products of the $p_{i}$ where each prime occurs at most once: If such a product $x$ does not contain precisely the primes $p_{i_{1}}, \ldots, p_{i_{r}}$ then $x$ is the greatest common divisor of $d_{i_{1}}, \ldots, d_{i_{r}}$. The set $Q$ has $2^{N}$ elements. 
Let $m \geq 1$ be an arbitrary integer. If $N=m$, there exists by Theorem 38 for every integer $n \geq 2 N+1=2 m+1$ a homotopy elliptic surface $W$ with $\chi_{h}(W)=n$ that has $2^{m}$ symplectic structures realizing all elements in $Q$ as the divisibility of their canonical classes. Since $d$ is odd, the 4-manifolds $W$ are nonspin.

If $N=2 m-1$, there exists for every even integer $n \geq 3 N+1=6 m-2$ a homotopy elliptic surface $W$ with $\chi_{h}(W)=n$ that has $2^{2 m-1}$ symplectic structures realizing all elements in $Q$ multiplied by 2 as the divisibility of their canonical classes. Since all divisibilities are even, the manifold $W$ is spin. If $N=2 m$, we can choose $n=6 m+2$ to get a spin homotopy elliptic surface $W$ with $\chi_{h}(W)=6 m+2$ and $2^{2 m}$ inequivalent symplectic structures.

We can extend construction in the proof of Theorem 38 to the spin manifolds in Theorem 19 with $c_{1}^{2}>0$ :

Theorem 41. Let $N \geq 1$ be an integer. Suppose that $d \geq 2$ is an even integer and $d_{0}, \ldots, d_{N}$ are positive even integers dividing $d$ as in Definition 37. Let $Q$ be the associated set of greatest common divisors. Let $m$ be an integer such that $2 m \geq 3 N+2$, and let $t \geq 1$ be an arbitrary integer. Then there exists a simply connected closed spin 4-manifold $W$ with invariants

$$
c_{1}^{2}(W)=2 t d^{2}, \quad e(W)=t d^{2}+24 m, \quad \sigma(W)=-16 m,
$$

and the property that for each integer $q \in Q$, the manifold $W$ admits a symplectic structure whose canonical class $K$ has divisibility equal to $q$. Hence $W$ admits at least $|Q|$ inequivalent symplectic structures.

Proof. Let $l=2 m-2 N$. By the construction of Theorem 19, there exists a simply connected symplectic spin 4-manifold $X$ with invariants

$$
c_{1}^{2}(X)=2 t d^{2}, \quad e(X)=t d^{2}+12 l, \quad \sigma(X)=-8 l, \quad K_{X}=d\left(\frac{1}{2} l F+R+t \Sigma_{M}\right) .
$$

In particular, the canonical class of $X$ has divisibility $d$. In the construction of $X$ starting from the elliptic surface $E(l)$, we have only used one Lagrangian rim torus. Hence $l-2$ of the $l-1$ triples of Lagrangian rim tori in $E(l)$ (see Example 30) remain unchanged. Note that $l-2 \geq N$ by our assumptions. Since the symplectic form on $E(l)$ in a neighbourhood of these tori does not change in the construction of $X$ by the Gompf fibre sum, we can assume that $X$ contains at least $N$ triples of Lagrangian tori as in the proof of Theorem 38. We can now use the same construction as in this theorem on the $N$ triples of Lagrangian tori in $X$ to get a simply connected spin 4-manifold $W$ with invariants

$$
\begin{aligned}
c_{1}^{2}(W)=2 t d^{2}, \quad e(W) & =t d^{2}+12 l+24 N \quad \sigma(W)=-8 l-16 N=-16 m, \\
& =t d^{2}+24 m,
\end{aligned}
$$


admitting $2^{N}$ symplectic structures. In particular, for each $q \in Q$ the manifold $W$ admits a symplectic structure $\omega_{I}$ whose canonical class is given by (6) and (7) if the term $d l F$ is replaced by $K_{X}=d\left(\frac{1}{2} l F+R+t \Sigma_{M}\right)$. It follows again that the canonical class of $\omega_{I}$ has divisibility precisely equal to $q$.

Corollary 42. Let $d \geq 6$ be an even integer, and let $t \geq 1$ and $m \geq 3$ be arbitrary integers. Then there exists a simply connected closed spin 4-manifold $W$ with invariants

$$
c_{1}^{2}(W)=2 t d^{2}, \quad e(W)=t d^{2}+24 m, \quad \sigma(W)=-16 m
$$

such that $W$ admits at least two inequivalent symplectic structures.

This follows with $N=1$ and choosing $d_{0}=d$ and $d_{1}=2$, since in this case $Q$ consists of two elements.

Example 43. We consider Corollary 42 for the spin homotopy Horikawa surfaces in Example 20. Let $t \geq 1$ and $k \geq 3$ be arbitrary odd integers, and define an integer $m$ by $2 m=3 t k^{2}+3$. Let $d=2 k$ and $d_{1}=2$. Since $d=2 k$ is not divisible by 4 , the set $Q$ is equal to $\{2 k, 2\}$ by Definition 37 . Hence there exists a spin homotopy Horikawa surface $X$ on the Noether line with invariants $c_{1}^{2}(X)=8 t k^{2}$ and $\chi_{h}(X)=4 t k^{2}+3$, and admitting two inequivalent symplectic structures: the canonical class of the first symplectic structure has divisibility $2 k$, while that of the second is divisible only by 2 .

Similarly, we can extend the construction in Theorem 41 to the nonspin manifolds in Theorem 22 with $c_{1}^{2}>0$ :

Theorem 44. Let $N \geq 1$ be an integer. Suppose $d \geq 3$ is an odd integer, and let $d_{0}, \ldots, d_{N}$ be positive integers dividing $d$ as in Definition 37. Let $Q$ be the associated set of greatest common divisors. Let $m \geq 2 N+2$ and $t \geq 1$ be arbitrary integers. Then there exists a simply connected closed nonspin 4-manifold $W$ with invariants

$$
c_{1}^{2}(W)=8 t d^{2}, \quad e(W)=4 t d^{2}+12 m, \quad \sigma(W)=-8 m
$$

and the property that for each integer $q \in Q$, the manifold $W$ admits a symplectic structure whose canonical class $K$ has divisibility equal to $q$. Hence $W$ admits at least $|Q|$ inequivalent symplectic structures.

Proof. The proof is analogous to the proof of Theorem 41. Let $l=m-N$. By the construction of Theorem 22, there exists a simply connected nonspin symplectic 4-manifold $X$ with invariants

$$
c_{1}^{2}(X)=8 t d^{2}, \quad e(X)=4 t d^{2}+12 l, \quad \sigma(X)=-8 l
$$


whose canonical class $K_{X}$ has divisibility $d$. The manifold $X$ contains $l-2$ triples of Lagrangian tori. By our assumptions, $l-2 \geq N$. Hence we can perform the construction in Theorem 38 (for $d$ odd) to get a simply connected nonspin 4-manifold $W$ with invariants

$$
\begin{aligned}
c_{1}^{2}(W)=8 t d^{2}, \quad e(W) & =4 t d^{2}+12 l+12 N \quad \sigma(X)=-8 l-8 N=-8 m . \\
& =4 t d^{2}+12 m,
\end{aligned}
$$

The 4-manifold $W$ admits for every integer $q \in Q$ a symplectic structure whose canonical class has divisibility equal to $q$.

Choosing $N=1, d_{0}=d$ and $d_{1}=1$, the set $Q$ contains two elements.

Corollary 45. Let $d \geq 3$ be an odd integer, and let $t \geq 1$ and $m \geq 4$ be integers. Then there exists a simply connected closed nonspin 4-manifold $W$ with invariants

$$
c_{1}^{2}(W)=8 t d^{2}, \quad e(W)=4 t d^{2}+12 m, \quad \sigma(W)=-8 m
$$

such that $W$ admits at least two inequivalent symplectic structures.

\section{Branched coverings}

Let $M^{n}$ be a closed, oriented smooth manifold, and let $F^{n-2}$ be a closed, oriented submanifold of codimension 2. Suppose the fundamental class $[F] \in H_{n-2}(M ; \mathbb{Z})$ is divisible by an integer $m>1$ and choose a class $B \in H_{n-2}(M ; \mathbb{Z})$ such that $[F]=m B$. The integer $m$ together with $B$ determine a branched covering of $M$.

Definition 46. We denote by $\phi: M(F, B, m) \rightarrow M$ the $m$-fold branched covering of $M$ branched over $F$ and determined by $m$ and $B$.

For the construction of branched coverings, see [Hirzebruch 1969]. The smooth manifold $M(F, B, m)$ has the properties that

- over the complement $M^{\prime}=M \backslash F$, the map $\phi: \phi^{-1}\left(M^{\prime}\right) \rightarrow M^{\prime}$ is a standard $m$-fold cyclic covering;

- $\phi$ maps the submanifold $\bar{F}=\phi^{-1}(F)$ diffeomorphically onto $F$, and on tubular neighbourhoods of $\bar{F}$ and $F$, the map $\phi: v(\bar{F}) \rightarrow v(F)$ is locally of the form

$$
U \times D^{2} \rightarrow U \times D^{2}, \quad(x, z) \mapsto\left(x, z^{m}\right),
$$

where $D^{2}$ is considered as the unit disk in $\mathbb{C}$.

Suppose $M$ is a smooth complex algebraic surface, and let $D \subset M$ be a smooth connected complex curve. If $m>0$ is an integer that divides $[D]$ and $B \in H_{2}(M ; \mathbb{Z})$ is a homology class such that $[D]=m B$, then the branched covering $M(D, B, m)$ also admits the structure of an algebraic surface. 
Proposition 47. Let $D$ be a smooth connected complex curve in a complex surface $M$ such that $[D]=m B$. Let $\phi: M(D, B, m) \rightarrow M$ be the branched covering. Then the invariants of $N:=M(D, B, m)$ are

(a) $K_{N}=\phi^{*}\left(K_{M}+(m-1) B\right)$,

(b) $c_{1}^{2}(N)=m\left(K_{M}+(m-1) B\right)^{2}$,

(c) $e(N)=m e(M)-(m-1) e(D)$,

where $e(D)=2-2 g(D)=-\left(K_{M} \cdot D+D^{2}\right)$ by the adjunction formula.

Proof. The formula for $e(N)$ follows by a well-known formula for the Euler characteristic of a topological space decomposed into two pieces and the formula for standard, unramified coverings. The formula for $c_{1}^{2}(N)$ then follows by the signature formula of Hirzebruch [1969]:

$$
\sigma(N)=m \sigma(M)-\frac{m^{2}-1}{3 m} D^{2} .
$$

The formula for $K_{N}$ can be found in [Barth et al. 1984, Chapter I, Lemma 17.1].

Suppose that the complex curve $D$ is contained in the linear system $\left|n K_{M}\right|$ and hence represents in homology a multiple $n K_{M}$ of the canonical class of $M$. Let $m>0$ be an integer dividing $n$ and write $n=m a$. Now set $[D]=n K_{M}$ and $B=a K_{M}$ in Proposition 47.

Corollary 48. Let $D$ be a smooth connected complex curve in a complex surface $M$ with $[D]=n K_{M}$ and $\phi: M\left(D, a K_{M}, m\right) \rightarrow M$ the branched covering. Then the invariants of $N:=M\left(D, a K_{M}, m\right)$ are

(a) $K_{N}=(n+1-a) \phi^{*} K_{M}$,

(b) $c_{1}^{2}(N)=m(n+1-a)^{2} c_{1}^{2}(M)$,

(c) $e(N)=m e(M)+(m-1) n(n+1) c_{1}^{2}(M)$.

We consider again the general situation that $M$ is a smooth, oriented manifold and $F$ is an oriented submanifold of codimension 2. The fundamental group of $M$ is related to the fundamental group of the complement $M^{\prime}=M \backslash F$ by

$$
\pi_{1}(M) \cong \pi_{1}\left(M^{\prime}\right) / N(\sigma)
$$

where $\sigma$ denotes the meridian to $F$, given by a circle fibre of $\partial v(F) \rightarrow F$, and $N(\sigma)$ denotes the normal subgroup in $\pi_{1}\left(M^{\prime}\right)$ generated by this element (a proof can be found in the appendix of [Hamilton 2008]). Using this formula, the fundamental group of a branched covering can be calculated in the following case. 
Theorem 49. Let $M^{n}$ be a closed oriented manifold, and let $F^{n-2}$ be a closed oriented submanifold. Suppose in addition that the fundamental group of $M^{\prime}$ is abelian. Then for all $m$ and $B$ with $[F]=m B$, there exists an isomorphism

$$
\pi_{1}(M(F, B, m)) \cong \pi_{1}(M) .
$$

Proof. Let $k>0$ denote the maximal integer dividing $[F]$. Since $m$ divides $k$, we can write $k=m a$ with $a>0$. Let $\bar{M}^{\prime}$ denote the complement to $\bar{F}$ in $M(F, B, m)$, and let $\bar{\sigma}$ be the meridian to $\bar{F}$. By Equation (8) we have

$$
\pi_{1}(M(F, B, m)) \cong \pi_{1}\left(\bar{M}^{\prime}\right) / N(\bar{\sigma}) .
$$

There is an exact sequence $0 \rightarrow \pi_{1}\left(\bar{M}^{\prime}\right) \stackrel{\pi_{*}}{\longrightarrow} \pi_{1}\left(M^{\prime}\right) \rightarrow \mathbb{Z}_{m} \rightarrow 0$ since $\pi: \bar{M}^{\prime} \rightarrow M^{\prime}$ is an $m$-fold cyclic covering. The assumption that $\pi_{1}\left(M^{\prime}\right)$ is abelian implies that $\pi_{1}\left(\bar{M}^{\prime}\right)$ is also abelian. Therefore the normal subgroups generated by the meridians are cyclic. The endpoints of the lifts of $0, \sigma, 2 \sigma, \ldots,(m-1) \sigma$, where $\sigma$ is the meridian to $F$, realize all $m$ points in the fibre over the basepoint. This implies that the induced map $\pi_{*}: \pi_{1}\left(\bar{M}^{\prime}\right) \longrightarrow \pi_{1}\left(M^{\prime}\right) /\langle\sigma\rangle$ is surjective. The kernel of this map is equal to $\langle\bar{\sigma}\rangle$, because only the multiples of $m \sigma=\pi_{*} \bar{\sigma}$ lift to loops in $\bar{M}^{\prime}$; hence

$$
\pi_{1}\left(\bar{M}^{\prime}\right) /\langle\bar{\sigma}\rangle \stackrel{\cong}{\longrightarrow} \pi_{1}\left(M^{\prime}\right) /\langle\sigma\rangle .
$$

Again by Equation (8), this implies $\pi_{1}(M(F, B, m)) \cong \pi_{1}(M)$.

We want to apply this theorem in the case where $M$ is a 4-manifold and $F$ is an embedded surface. Even if $M$ is simply connected, the complement $M^{\prime}$ does not have abelian fundamental group in general. However, in the complex case, we can use the following, which is [Nori 1983, Proposition 3.27].

Theorem 50. Let $M$ be a smooth complex algebraic surface, and let $D, E \subset M$ be smooth complex curves that intersect transversely. Assume that $D^{\prime 2}>0$ for every connected component $D^{\prime} \subset D$. Then the kernel of $\pi_{1}(M \backslash(D \cup E)) \rightarrow \pi_{1}(M \backslash E)$ is a finitely generated abelian group.

If $E=\varnothing$, this implies that the kernel of $\pi_{1}\left(M^{\prime}\right) \rightarrow \pi_{1}(M)$ is a finitely generated abelian group if $D$ is connected and $D^{2}>0$, where $M^{\prime}=M \backslash D$. If $M$ is simply connected, it follows that $\pi_{1}\left(M^{\prime}\right)$ is abelian. Thus with Theorem 49 we get this:

Corollary 51. Let $M$ be a simply connected, smooth complex algebraic surface, and let $D \subset M$ be a smooth connected complex curve with $D^{2}>0$. Let $\bar{M}$ be a cyclic ramified cover of $M$ branched over $D$. Then $\bar{M}$ is also simply connected.

Catanese [1984] has also used in a different situation restrictions on divisors to ensure that certain ramified coverings are simply connected. 


\section{Surfaces of general type and pluricanonical systems}

We collect some results concerning the geography of simply connected surfaces of general type and the existence of smooth divisors in pluricanonical systems.

The following is the main geography result we use for our constructions.

Theorem 52 [Persson 1981, Proposition 3.23]. Let $x, y$ be positive integers such that $2 x-6 \leq y \leq 4 x-8$. Then there exists a simply connected minimal complex surface $M$ of general type such that $\chi_{h}(M)=x$ and $c_{1}^{2}(M)=y$. Furthermore, $M$ can be chosen as a genus 2 fibration.

The smallest integer $x$ for which an inequality can be realized with $y>0$ is $x=3$. Since $\chi_{h}(M)=p_{g}(M)+1$ for simply connected surfaces, this corresponds to surfaces with $p_{g}=2$. Hence from Theorem 52, we get minimal simply connected complex surfaces $M$ with $p_{g}=2$ and $K^{2}=1,2,3,4$. Similarly for $x=4$ we get surfaces with $p_{g}=3$ and $K^{2}=2, \ldots, 8$.

Proposition 53. For $K^{2}=1$ and $K^{2}=2$, all possible values for $p_{g}$ given by the Noether inequality $K^{2} \geq 2 p_{g}-4$ can be realized by simply connected minimal complex surfaces of general type.

Proof. By the Noether inequality, the only possible values for $p_{g}$ are $p_{g}=0,1,2$ if $K^{2}=1$ and $p_{g}=0,1,2,3$ if $K^{2}=2$. The cases $p_{g}=2$ for $K^{2}=1$ and $p_{g}=2,3$ for $K^{2}=2$ are covered by Persson's theorem. In particular, the surface with $K^{2}=1$ and $p_{g}=2$ and the surface with $K^{2}=2$ and $p_{g}=3$ are Horikawa surfaces described in [Horikawa 1976a; 1976b]. The remaining cases can also be covered: The Barlow surface from [1985] is a simply connected numerical Godeaux surface, that is, a minimal complex surface of general type with $K^{2}=1$ and $p_{g}=0$. Simply connected minimal surfaces of general type with $K^{2}=1,2$ and $p_{g}=1$ exist by constructions due to Enriques; see [Catanese 1979; Catanese and Debarre 1989; Chakiris 1980]. Finally, Lee and Park [2007] have constructed a simply connected minimal surface of general type with $K^{2}=2$ and $p_{g}=0$. It is a numerical Campedelli surface.

Suppose $M$ is a minimal smooth complex algebraic surface of general type and consider the multiples $L=n K=K^{\otimes n}$ of the canonical line bundle of $M$. By a theorem of Bombieri [Bombieri 1973; Barth et al. 1984], all divisors in the linear system $|n K|$ are connected. If $|n K|$ has no fixed parts and is base point free, it determines an everywhere-defined holomorphic map to a projective space, and we can find a nonsingular divisor representing $n K$ by taking the preimage of a generic hyperplane section.

Theorem 54. Let $M$ be a minimal smooth complex algebraic surface of general type. Then the pluricanonical system $|n K|$ determines an everywhere defined holomorphic map in the cases 
- $n \geq 4$,

- $n=3$ and $K^{2} \geq 2$, and

- $n=2$ and $K^{2} \geq 5$ or $p_{g} \geq 1$.

For proofs and references, see [Bombieri 1970; 1973; Catanese and Tovena 1992; Kodaira 1968; Mendes Lopes and Pardini 2002; Reider 1988].

Remark 55. In some of the remaining cases it is also known that pluricanonical systems define a holomorphic map. In particular, suppose that $M$ is a numerical Godeaux surface. Then the map defined by $|3 K|$ is holomorphic if $H_{1}(M ; \mathbb{Z})=0$ or $\mathbb{Z}_{2}$, for example, if $M$ is simply connected [Miyaoka 1976; Reid 1978]. This is also known for the map defined by $|2 K|$ in the case of a simply connected surface $M$ with $K^{2}=4$ and $p_{g}=0$ by [Catanese and Tovena 1992; Kotschick 1994].

\section{Branched covering construction of algebraic surfaces with divisible canonical class}

Suppose that $M$ is a simply connected minimal complex surface of general type. Let $m, d \geq 2$ be integers such that $m-1$ divides $d-1$ and define the integers $a=(d-1) /(m-1)$ and $n=m a$. Then $d=n+1-a$ and the assumptions imply that $n \geq 2$. We assume in addition that $n K_{M}$ can be represented by a smooth complex connected curve $D$ in $M$; see Theorem 54 . Let $\bar{M}=M\left(D, a K_{M}, m\right)$ denote the associated $m$-fold branched cover over the curve $D$.

Theorem 56. Let $M$ be a simply connected minimal surface of general type, and let $m, d \geq 2$ be integers such that $d-1$ is divisible by $m-1$ with quotient $a$. Suppose that $D$ is a smooth connected curve in the linear system $\left|n K_{M}\right|$, where $n=m a$. Then the $m$-fold cover of $M$, branched over $D$, is a simply connected complex surface $\bar{M}$ of general type with invariants

$$
\begin{array}{rlrl}
K_{\bar{M}} & =d \phi^{*} K_{M}, & e(\bar{M}) & =m\left(e(M)+(d-1)(d+a) c_{1}^{2}(M)\right), \\
c_{1}^{2}(\bar{M}) & =m d^{2} c_{1}^{2}(M), \quad \chi_{h}(\bar{M})=m \chi_{h}(M)+\frac{1}{12} m(d-1)(2 d+a+1) c_{1}^{2}(M), \\
\sigma(\bar{M}) & =-\frac{1}{3} m\left(2 e(M)+(d(d-2)+2 a(d-1)) c_{1}^{2}(M)\right) .
\end{array}
$$

In particular, the canonical class $K_{\bar{M}}$ is divisible by $d$ and $\bar{M}$ is minimal.

Proof. The invariants are given by Corollary 48. Since $D^{2}=n^{2} K_{M}^{2}>0$, the complex surface $\bar{M}$ is simply connected by Corollary 51 . Also, $\bar{M}$ is of general type because $c_{1}^{2}(\bar{M})>0$ and $\bar{M}$ cannot be rational or ruled. Minimality follows from Lemma 2 , since the divisibility of $K_{\bar{M}}$ is at least $d \geq 2$.

Note that the signature $\sigma(\bar{M})$ is always negative; hence surfaces with positive signature cannot be constructed in this way. 
The transformation

$$
\Phi:\left(e(M), c_{1}^{2}(M)\right) \mapsto\left(e(\bar{M}), c_{1}^{2}(\bar{M})\right)
$$

given by Theorem 56 is linear and can be written as

$$
\left(\begin{array}{c}
e(\bar{M}) \\
c_{1}^{2}(\bar{M})
\end{array}\right)=m\left(\begin{array}{cc}
1 & \Delta \\
0 & d^{2}
\end{array}\right)\left(\begin{array}{c}
e(M) \\
c_{1}^{2}(M)
\end{array}\right),
$$

with the abbreviation $\Delta=(d-1)(d+a)$. This map is invertible over $\mathbb{R}$ and maps the quadrant $\mathbb{R}^{+} \times \mathbb{R}^{+}$of positive coordinates in $\mathbb{R} \times \mathbb{R}$ into the same quadrant. The inverse of $\Phi$ is given by

$$
\left(\begin{array}{c}
e(M) \\
c_{1}^{2}(M)
\end{array}\right)=\frac{1}{m}\left(\begin{array}{cc}
1 & -\Delta / d^{2} \\
0 & 1 / d^{2}
\end{array}\right)\left(\begin{array}{c}
e(\bar{M}) \\
c_{1}^{2}(\bar{M})
\end{array}\right)
$$

Definition 57. We call a point in $\mathbb{R}^{+} \times \mathbb{R}^{+}$admissible if $e(M)+c_{1}^{2}(M) \equiv 0 \bmod 12$. The coordinates $e(M)$ and $c_{1}^{2}(M)$ of a complex surface are always admissible by the Noether formula.

Lemma 58. The image of the admissible points in $\mathbb{R}^{+} \times \mathbb{R}^{+}$under the map $\Phi$ consists of the points satisfying

$e(\bar{M}) \equiv 0 \bmod m, \quad c_{1}^{2}(\bar{M}) \equiv 0 \bmod m d^{2}, \quad \frac{1}{m} e(\bar{M})+\frac{1-\Delta}{m d^{2}} c_{1}^{2}(\bar{M}) \equiv 0 \bmod 12$.

The proof is immediate by the formula for the inverse of $\Phi$. We want to calculate the image under $\Phi$ of the sector given by Theorem 52. First, we rewrite Persson's theorem in an equivalent form (we omit the proof):

Corollary 59. Let e and $c$ be positive integers with $c \geq 36-e$ and $e+c \equiv 0 \bmod 12$. If $\frac{1}{5}(e-36) \leq c \leq \frac{1}{2}(e-24)$, then there exists a simply connected minimal surface $M$ of general type with invariants $e(M)=e$ and $c_{1}^{2}(M)=c$.

In the next step, we calculate the image under $\Phi$ of the lines in the $(e, c)$-plane that appear in this corollary. A short calculation shows that the line $c=\frac{1}{5}(e-36)$ maps to

$$
c_{1}^{2}(\bar{M})=\frac{d^{2}}{5+\Delta}(e(\bar{M})-36 m),
$$

while the line $c=\frac{1}{2}(e-24)$ maps to

$$
c_{1}^{2}(\bar{M})=\frac{d^{2}}{2+\Delta}(e(\bar{M})-24 m) .
$$

Similarly, the constraint $c \geq 36-e$ maps to

$$
c_{1}^{2}(\bar{M}) \leq \frac{d^{2}}{-1+\Delta}(e(\bar{M})-36 m) .
$$


It follows that the image under $\Phi$ of the lattice points given by the constraints in Corollary 59 consists precisely of those points in the sector between the lines (9) and (10) that satisfy the constraint (11) and the constraints in Lemma 58.

The surfaces in Theorem 52 satisfy $p_{g} \geq 2$ and $K^{2} \geq 1$. By Theorem 54, the linear system $|n K|$ for $n \geq 2$ on these surfaces defines a holomorphic map, except possibly in the case $p_{g}=2, K^{2}=1$ and $n=3$. Since $n=m a$ and $m \geq 2$, this occurs only for $m=3, a=1$ and $d=3$. The corresponding image under $\Phi$ has invariants $\left(e, c_{1}^{2}\right)=(129,27)$. This exception is implicitly understood in the following theorem. In all other cases we can consider the branched covering construction above. This can be summarized as follows: Consider integers $m, a, d$ as above, with $m, d \geq 2, a \geq 1$ and $\Delta=(d-1)(d+a)$.

Theorem 60. Let $x$ and $y$ be positive integers such that $y(1-\Delta) \geq 36-x$ and $x+(1-\Delta) y \equiv 0 \bmod 12$. If

$$
\frac{1}{(5+\Delta)}(x-36) \leq y \leq \frac{1}{(2+\Delta)}(x-24) \text {, }
$$

then there is a simply connected minimal complex surface $\bar{M}$ of general type with invariants $e(\bar{M})=m x$ and $c_{1}^{2}(\bar{M})=m d^{2} y$ such that the canonical class of $\bar{M}$ is divisible by $d$.

We calculate some explicit examples for the branched covering construction given by Theorem 60 and for some surfaces not covered by Persson's theorem. For any $d \geq 2$, we can choose $m=2$ and $a=d-1$, corresponding to 2-fold covers branched over $(2 d-2) K$. The formulas for the invariants simplify to

$$
\begin{aligned}
c_{1}^{2}(\bar{M})=2 d^{2} c_{1}^{2}(M), \quad e(\bar{M}) & =24 \chi_{h}(M)+2 d(2 d-3) c_{1}^{2}(M), \\
\chi_{h}(\bar{M}) & =2 \chi_{h}(M)+\frac{1}{2} d(d-1) c_{1}^{2}(M) .
\end{aligned}
$$

The first two examples are double coverings with $m=2$, whereas the third example uses coverings of higher degree. Because of their topological invariants, some of the surfaces are homeomorphic by Freedman's theorem to the simply connected symplectic 4-manifolds constructed in Sections 6 and 7.

Example 61. We consider the Horikawa surfaces [1976a] on the Noether line $c_{1}^{2}=2 \chi_{h}-6$, which exist for every $\chi_{h} \geq 4$ and are also given by Persson's Theorem 52. In this case $p_{g} \geq 3$ and $c_{1}^{2} \geq 2$; hence by Theorem 54, the linear system $|n K|$ for $n \geq 2$ defines a holomorphic map on these surfaces.

Proposition 62. Let $M$ be a Horikawa surface on the Noether line $c_{1}^{2}=2 \chi_{h}-6$, where $\chi_{h}=4+l$ for $l \geq 0$. Then the 2 -fold cover $\bar{M}$ of the surface $M$, branched 
over $(2 d-2) K_{M}$ for an integer $d \geq 2$, has invariants

$$
\begin{aligned}
c_{1}^{2}(\bar{M}) & =4 d^{2}(l+1), & \chi_{h}(\bar{M}) & =6+(2+d(d-1))(l+1), \\
e(\bar{M}) & =72+4(l+1)\left(6+2 d^{2}-3 d\right), & \sigma(\bar{M}) & =-48-4(l+1)\left(4+d^{2}-2 d\right) .
\end{aligned}
$$

The canonical class $K_{\bar{M}}$ is divisible by $d$.

For $d$ even, the integer $d^{2}-2 d=d(d-2)$ is divisible by 4 ; hence $\sigma$ is indeed divisible by 16 , which is necessary by Rohlin's theorem. The invariants are on the line

$$
c_{1}^{2}(\bar{M})=\frac{4 d^{2}}{2+d(d-1)}\left(\chi_{h}(\bar{M})-6\right),
$$

which has inclination close to 4 for $d$ very large.

Example 63. We calculate the invariants for the branched covers with $m=2$ and integers $d \geq 3$ for the surfaces given by Proposition 53. Since $n=m a \geq 4$ in this case, Theorem 54 shows that the linear system $|n K|$ defines a holomorphic map and we can use the branched covering construction.

Proposition 64. Let $M$ be a minimal complex surface of general type with $K^{2}$ equal to 1 or 2 . The 2-fold cover $\bar{M}$ of the surface $M$, branched over $(2 d-2) K_{M}$ for an integer $d \geq 3$, has invariants as follows:

$$
\begin{aligned}
& \text { If } K^{2}=1 \text { and } p_{g}=0,1,2, \quad c_{1}^{2}(\bar{M})=2 d^{2}, \\
& e(\bar{M})=24\left(p_{g}+1\right)+2 d(2 d-3) \text {, } \\
& \sigma(\bar{M})=-16\left(p_{g}+1\right)-2 d(d-2) \text {. } \\
& \text { If } K^{2}=2 \text { and } p_{g}=0,1,2,3, \quad c_{1}^{2}(\bar{M})=4 d^{2} \text {, } \\
& e(\bar{M})=24\left(p_{g}+1\right)+4 d(2 d-3) \text {, } \\
& \sigma(\bar{M})=-16\left(p_{g}+1\right)-4 d(d-2) .
\end{aligned}
$$

In both cases the canonical class $K_{\bar{M}}$ is divisible by $d$.

Example 65. Consider the Barlow surface $M_{\mathrm{B}}$ and the surface $M_{\mathrm{LP}}$ of Lee and Park mentioned in the proof of Proposition 53. The invariants are

$$
\begin{aligned}
c_{1}^{2}\left(M_{\mathrm{B}}\right) & =1, \quad \chi_{h}\left(M_{\mathrm{B}}\right)=1, & e\left(M_{\mathrm{B}}\right)=11 ; \\
c_{1}^{2}\left(M_{\mathrm{LP}}\right) & =2, \quad \chi_{h}\left(M_{\mathrm{LP}}\right)=1, & e\left(M_{\mathrm{LP}}\right)=10 .
\end{aligned}
$$

By Theorem 54, we can consider branched covers over both surfaces with $m a \geq 3$ (the Barlow surface is a simply connected numerical Godeaux surface, and hence $|3 K|$ defines a holomorphic map by Remark 55). See Tables 1 and 2 for a calculation of the invariants of $\bar{M}$ for small values of $d$ and $m$. There is an agreement between the 4-fold cover of $M_{\mathrm{B}}$ branched over $4 K_{M}$ and the 2-fold cover of $M_{\mathrm{LP}}$ branched over $6 K_{M}$ : Both have the same Chern invariants and the same divisibility 


\begin{tabular}{ccccccccc}
\hline$d$ & $m$ & $m a$ & $(d-1)(d+a)$ & $e\left(\overline{M_{\mathrm{B}}}\right)$ & $c_{1}^{2}\left(\overline{M_{\mathrm{B}}}\right)$ & $\chi_{h}\left(\overline{M_{\mathrm{B}}}\right)$ & $b_{2}^{+}\left(\overline{M_{\mathrm{B}}}\right)$ & $\sigma\left(\overline{M_{\mathrm{B}}}\right)$ \\
\hline 3 & 2 & 4 & 10 & 42 & 18 & 5 & 9 & -22 \\
3 & 3 & 3 & 8 & 57 & 27 & 7 & 13 & -29 \\
4 & 2 & 6 & 21 & 64 & 32 & 8 & 15 & -32 \\
4 & 4 & 4 & 15 & 104 & 64 & 14 & 27 & -48 \\
5 & 2 & 8 & 36 & 94 & 50 & 12 & 23 & -46 \\
5 & 3 & 6 & 28 & 117 & 75 & 16 & 31 & -53 \\
5 & 5 & 5 & 24 & 175 & 125 & 25 & 49 & -75 \\
6 & 2 & 10 & 55 & 132 & 72 & 17 & 33 & -64 \\
6 & 6 & 6 & 35 & 276 & 216 & 41 & 81 & -112 \\
\hline
\end{tabular}

Table 1. Ramified coverings of the Barlow surface $M_{\mathrm{B}}$ of degree $m$ branched over $m a K$.

\begin{tabular}{ccccccccc}
\hline$d$ & $m$ & $m a$ & $(d-1)(d+a)$ & $e\left(\overline{M_{\mathrm{LP}}}\right)$ & $c_{1}^{2}\left(\overline{M_{\mathrm{LP}}}\right)$ & $\chi_{h}\left(\overline{M_{\mathrm{LP}}}\right)$ & $b_{2}^{+}\left(\overline{M_{\mathrm{LP}}}\right)$ & $\sigma\left(\overline{M_{\mathrm{LP}}}\right)$ \\
\hline 3 & 2 & 4 & 10 & 60 & 36 & 8 & 15 & -28 \\
3 & 3 & 3 & 8 & 78 & 54 & 11 & 21 & -34 \\
4 & 2 & 6 & 21 & 104 & 64 & 14 & 27 & -48 \\
4 & 4 & 4 & 15 & 160 & 128 & 24 & 47 & -64 \\
5 & 2 & 8 & 36 & 164 & 100 & 22 & 43 & -76 \\
5 & 3 & 6 & 28 & 198 & 150 & 29 & 57 & -82 \\
5 & 5 & 5 & 24 & 290 & 250 & 45 & 89 & -110 \\
6 & 2 & 10 & 55 & 240 & 144 & 32 & 63 & -112 \\
6 & 6 & 6 & 35 & 480 & 432 & 76 & 151 & -176 \\
\hline
\end{tabular}

Table 2. Ramified coverings of the Lee-Park surface $M_{\mathrm{LP}}$ of degree $m$ branched over $m a K$.

$d=4$ of the canonical class. Hence the manifolds are homeomorphic and by Theorem 4, both branched coverings have the same Seiberg-Witten invariants.

Remark 66. More general examples are possible by considering branched coverings over singular complex curves. The following example is described for instance in [Gompf and Stipsicz 1999, Chapter 7]: Let $B_{n, m}$ denote the singular complex curve in $\mathbb{C} P^{l} \times \mathbb{C} P^{l}$ that is the union of $2 n$ parallel copies of the first factor and $2 m$ parallel copies of the second factor. The curve $B_{n, m}$ represents in cohomology the class $2 n S_{1}+2 m S_{2}$, where $S_{1}=\left[\mathbb{C} P^{l} \times\{*\}\right]$ and $S_{2}=\left[\{*\} \times \mathbb{C} P^{l}\right]$. Let $X^{\prime}(n, m)$ denote the double covering of $\mathbb{C} P^{l} \times \mathbb{C} P^{l}$ branched over $B_{n, m}$. It is a singular complex surface that has a canonical resolution $X(n, m)$; see [Barth et al. 1984, Chapter III]. As a smooth 4-manifold, $X(n, m)$ is diffeomorphic to the double 
cover of $\mathbb{C} P^{l} \times \mathbb{C} P^{l}$ branched over the smooth curve $\widetilde{B}_{n, m}$ given by smoothing the double points. Hence the topological invariants for $X=X(n, m)$ can be calculated with the formulas from Proposition 47 to be

$$
c_{1}^{2}(X)=4(n-2)(m-2), \quad e(X)=6+2(2 m-1)(2 n-1), \quad \sigma(X)=-4 m n .
$$

Writing $X^{\prime}=X^{\prime}(n, m)$ and $M=\mathbb{C} P^{l} \times \mathbb{C} P^{l}$, denote by $\phi: X^{\prime} \rightarrow M$ the double covering, by $\pi: X \rightarrow X^{\prime}$ the canonical resolution, and by $\psi=\phi \circ \pi$ the composition. Since all singularities of $B_{n, m}$ are ordinary double points, $K_{X}$ can be calculated by a formula in [Barth et al. 1984, Theorem 7.2, Chapter III] as

$$
\begin{aligned}
K_{X}=\psi^{*}\left(K_{M}+\frac{1}{2} B_{m, n}\right) & =\psi^{*}\left(-2 S_{1}-2 S_{2}+n S_{1}+m S_{2}\right) \\
& =\psi^{*}\left((n-2) S_{1}+(m-2) S_{2}\right) .
\end{aligned}
$$

We interpret this formula as follows: The map $\psi: X \rightarrow \mathbb{C} P^{l} \times \mathbb{C} P^{l}$ followed by the projection onto the first factor defines a fibration $X \rightarrow \mathbb{C} P^{l}$ whose fibres are the branched covers of the rational curves $\{p\} \times \mathbb{C} P^{l}$, where $p \in \mathbb{C} P^{l}$. The generic rational curve among them is disjoint from the $2 m$ curves in $B_{n, m}$ parallel to $\{*\} \times \mathbb{C} P^{l}$ and intersects the $2 n$ curves parallel to $\mathbb{C} P^{l} \times\{*\}$ at $2 n$ points. This implies that the generic fibre $F_{2}$ of the fibration is a double branched cover of $\mathbb{C} P^{l}$ at $2 n$ distinct points and hence a smooth complex curve of genus $n-1$. This curve represents the class $\psi^{*} S_{2}$ in the surface $X$. Similarly, there is a fibration $X \rightarrow \mathbb{C} P^{l}$ in genus $m-1$ curves that represents $F_{1}=\psi^{*} S_{1}$. Hence we can write $K_{X}=(n-2) F_{1}+(m-2) F_{2}$. In particular, the divisibility of $K_{X}$ is the greatest common divisor of $n-2$ and $m-2$.

Remark 67. In [Catanese 1984; 1986; Catanese and Wajnryb 2007], the authors constructed certain families of simply connected surfaces of general type with divisible canonical class, using branched coverings over singular curves. Some of these surfaces are diffeomorphic but not deformation equivalent, thus giving counterexamples to a well-known conjecture.

\section{Acknowledgment}

This article is part of my thesis, submitted in May 2008 at the University of Munich. I would like to thank my advisor D. Kotschick.

\section{References}

[Auroux et al. 2005] D. Auroux, V. Muñoz, and F. Presas, "Lagrangian submanifolds and Lefschetz pencils”, J. Symplectic Geom. 3:2 (2005), 171-219. MR 2006i:53118 Zbl 1093.53085

[Barlow 1985] R. Barlow, "A simply connected surface of general type with $p_{g}=0$ ", Invent. Math. 79:2 (1985), 293-301. MR 87a:14033 Zbl 0561.14015 
[Barth et al. 1984] W. Barth, C. Peters, and A. Van de Ven, Compact complex surfaces, Ergebnisse series (3) 4, Springer, Berlin, 1984. MR 86c:32026 Zbl 0718.14023

[Bombieri 1970] E. Bombieri, "The pluricanonical map of a complex surface”, pp. 35-87 in Several Complex Variables, I (College Park, MD, 1970), edited by J. Horváth, Springer, Berlin, 1970. MR 43 \#1975 Zbl 0213.47601

[Bombieri 1973] E. Bombieri, "Canonical models of surfaces of general type", Inst. Hautes Études Sci. Publ. Math. 42 (1973), 171-219. MR 47 \#6710 Zbl 0259.14005

[Catanese 1979] F. Catanese, "Surfaces with $K^{2}=p_{g}=1$ and their period mapping", pp. 1-29 in Algebraic geometry (Copenhagen, 1978), edited by K. Lønsted, Lecture Notes in Math. 732, Springer, Berlin, 1979. MR 81c:14018 Zbl 0423.14019

[Catanese 1984] F. Catanese, "On the moduli spaces of surfaces of general type", J. Differential Geom. 19:2 (1984), 483-515. MR 86h:14031 Zbl 0549.14012

[Catanese 1986] F. Catanese, "Connected components of moduli spaces”, J. Differential Geom. 24:3 (1986), 395-399. MR 87m:14036 Zbl 0621.14014

[Catanese and Debarre 1989] F. Catanese and O. Debarre, "Surfaces with $K^{2}=2, p_{g}=1, q=0$ ", J. Reine Angew. Math. 395 (1989), 1-55. MR 89m:14017 Zbl 0658.14016

[Catanese and Tovena 1992] F. Catanese and F. Tovena, "Vector bundles, linear systems and extensions of $\pi_{1}$ ", pp. 51-70 in Complex algebraic varieties (Bayreuth, 1990), edited by K. Hulek et al., Lecture Notes in Math. 1507, Springer, Berlin, 1992. MR 93h:14028 Zbl 0788.14013

[Catanese and Wajnryb 2007] F. Catanese and B. Wajnryb, "Diffeomorphism of simply connected algebraic surfaces”, J. Differential Geom. 76:2 (2007), 177-213. MR 2008c:14057 Zbl 1127.14039

[Chakiris 1980] K. N. Chakiris, "Counterexamples to global Torelli for certain simply connected surfaces”, Bull. Amer. Math. Soc. (N.S.) 2:2 (1980), 297-299. MR 81d:14021 Zbl 0432.32014

[Chen 1987] Z. J. Chen, "On the geography of surfaces: Simply connected minimal surfaces with positive index”, Math. Ann. 277:1 (1987), 141-164. MR 88c:14057 Zbl 0595.14027

[Fintushel and Stern 1994] R. Fintushel and R. J. Stern, "Surgery in cusp neighborhoods and the geography of irreducible 4-manifolds", Invent. Math. 117:3 (1994), 455-523. MR 95f:57040 Zbl 0843.57021

[Fintushel and Stern 1997] R. Fintushel and R. J. Stern, "Rational blowdowns of smooth 4-manifolds", J. Differential Geom. 46:2 (1997), 181-235. MR 98j:57047 Zbl 0896.57022

[Fintushel and Stern 1998] R. Fintushel and R. J. Stern, "Knots, links, and 4-manifolds", Invent. Math. 134:2 (1998), 363-400. MR 99j:57033 Zbl 0914.57015

[Fintushel and Stern 2001] R. Fintushel and R. Stern, "The canonical class of a symplectic 4manifold", Turkish J. Math. 25:1 (2001), 137-145. MR 2002g:57053 Zbl 0977.57032

[Fintushel and Stern 2004] R. Fintushel and R. J. Stern, "Families of simply connected 4-manifolds with the same Seiberg-Witten invariants", Topology 43:6 (2004), 1449-1467. MR 2005d:57044 Zbl 1064.57036

[Freedman 1982] M. H. Freedman, "The topology of four-dimensional manifolds", J. Differential Geom. 17:3 (1982), 357-453. MR 84b:57006 Zbl 0528.57011

[Friedman and Morgan 1997] R. Friedman and J. W. Morgan, "Algebraic surfaces and SeibergWitten invariants”, J. Algebraic Geom. 6:3 (1997), 445-479. MR 99b:32045 Zbl 0896.14015

[Gompf 1991] R. E. Gompf, "Nuclei of elliptic surfaces", Topology 30:3 (1991), 479-511. MR 92f: $57042 \mathrm{Zbl} 0732.57010$

[Gompf 1995] R. E. Gompf, "A new construction of symplectic manifolds”, Ann. of Math. (2) 142:3 (1995), 527-595. MR 96j:57025 Zbl 0849.53027 
[Gompf and Mrowka 1993] R. E. Gompf and T. S. Mrowka, "Irreducible 4-manifolds need not be complex", Ann. of Math. (2) 138:1 (1993), 61-111. MR 95c:57053 Zbl 0805.57012

[Gompf and Stipsicz 1999] R. E. Gompf and A. I. Stipsicz, 4-manifolds and Kirby calculus, Graduate Studies in Mathematics 20, American Mathematical Society, Providence, RI, 1999. MR 2000h: 57038 Zbl 0933.57020

[Hamilton 2008] M. J. D. Hamilton, On symplectic 4-manifolds and contact 5-manifolds, thesis, Ludwig-Maximilians-Universität München, 2008, available at http://nbn-resolving.de/urn:nbn:de: bvb:19-87797.

[Hamilton and Kotschick 2006] M. J. D. Hamilton and D. Kotschick, "Minimality and irreducibility of symplectic four-manifolds", Int. Math. Res. Not. (2006), Art. ID 35032. MR 2007i:57023 Zbl 1101.53052

[Hirzebruch 1969] F. Hirzebruch, "The signature of ramified coverings", pp. 253-265 in Global Analysis (Papers in Honor of K. Kodaira), Univ. Tokyo Press, 1969. MR 41 \#2707 Zbl 0208.51802 [Horikawa 1976a] E. Horikawa, "Algebraic surfaces of general type with small $C_{1}^{2}$, I", Ann. of Math. (2) 104:2 (1976), 357-387. MR 54 \#12789 Zbl 0339.14024

[Horikawa 1976b] E. Horikawa, "Algebraic surfaces of general type with small $c_{1}^{2}$, II", Invent. Math. 37:2 (1976), 121-155. MR 57 \#334 Zbl 0339.14025

[Ionel and Parker 2004] E.-N. Ionel and T. H. Parker, "The symplectic sum formula for GromovWitten invariants”, Ann. of Math. (2) 159:3 (2004), 935-1025. MR 2006b:53110 Zbl 1075.53092

[Kodaira 1968] K. Kodaira, "Pluricanonical systems on algebraic surfaces of general type", J. Math. Soc. Japan 20 (1968), 170-192. MR 37 \#212 Zbl 0157.27704

[Kotschick 1994] D. Kotschick, "On the pluricanonical maps of Godeaux and Campedelli surfaces", Internat. J. Math. 5:1 (1994), 53-60. MR 94m:14053 Zbl 0810.14016

[Kotschick 1997] D. Kotschick, "The Seiberg-Witten invariants of symplectic four-manifolds", pp. 195-220 in Séminaire Bourbaki 1995/96 (Exposé 812), Astérisque 241, Société Mathématique de France, Paris, 1997. MR 98h:57057 Zbl 0882.57026

[Lee and Park 2007] Y. Lee and J. Park, "A simply connected surface of general type with $p_{g}=0$ and $K^{2}=2$ ", Invent. Math. 170:3 (2007), 483-505. MR 2008m:14076 Zbl 1126.14049

[Li and Liu 2001] T.-J. Li and A.-K. Liu, "Uniqueness of symplectic canonical class, surface cone and symplectic cone of 4-manifolds with $B^{+}=1$ ", J. Differential Geom. 58:2 (2001), 331-370. MR 2003g:57042 Zbl 1051.57035

[Liu 1996] A.-K. Liu, "Some new applications of general wall crossing formula, Gompf's conjecture and its applications", Math. Res. Lett. 3:5 (1996), 569-585. MR 97k:57038 Zbl 0872.57025

[McCarthy and Wolfson 1994] J. D. McCarthy and J. G. Wolfson, "Symplectic normal connect sum”, Topology 33:4 (1994), 729-764. MR 95h:57038 Zbl 0812.53033

[McMullen and Taubes 1999] C. T. McMullen and C. H. Taubes, "4-manifolds with inequivalent symplectic forms and 3-manifolds with inequivalent fibrations", Math. Res. Lett. 6:5-6 (1999), 681696. MR 2000m:57045 Zbl 0964.53051

[Mendes Lopes and Pardini 2002] M. Mendes Lopes and R. Pardini, "A survey on the bicanonical map of surfaces with $p_{g}=0$ and $K^{2} \geq 2$ ", pp. 277-287 in Algebraic geometry, edited by M. C. Beltrametti et al., de Gruyter, Berlin, 2002. MR 2003k:14051 Zbl 1046.14018

[Milnor and Stasheff 1974] J. W. Milnor and J. D. Stasheff, Characteristic classes, Annals of Mathematics Studies 76, Princeton University Press, 1974. MR 55 \#13428 Zbl 0298.57008

[Miyaoka 1976] Y. Miyaoka, "Tricanonical maps of numerical Godeaux surfaces", Invent. Math. 34:2 (1976), 99-111. MR 53 \#13236 Zbl 0337.14010 
[Morgan 1996] J. W. Morgan, The Seiberg-Witten equations and applications to the topology of smooth four-manifolds, Mathematical Notes 44, Princeton University Press, 1996. MR 97d:57042 Zbl 0846.57001

[Nori 1983] M. V. Nori, “Zariski's conjecture and related problems”, Ann. Sci. École Norm. Sup. (4) 16:2 (1983), 305-344. MR 86d:14027 Zbl 0527.14016

[Park 1998] J. Park, "The geography of irreducible 4-manifolds", Proc. Amer. Math. Soc. 126:8 (1998), 2493-2503. MR 98j:57034 Zbl 0895.57005

[Park 2002] J. Park, "The geography of Spin symplectic 4-manifolds", Math. Z. 240:2 (2002), 405421. MR 2003c:57030 Zbl 1030.57032

[Park and Szabó 2000] B. D. Park and Z. Szabó, "The geography problem for irreducible spin fourmanifolds", Trans. Amer. Math. Soc. 352:8 (2000), 3639-3650. MR 2000m:57037 Zbl 0947.57023

[Persson 1981] U. Persson, "Chern invariants of surfaces of general type", Compositio Math. 43:1 (1981), 3-58. MR 83b:14012 Zbl 0479.14018

[Persson et al. 1996] U. Persson, C. Peters, and G. Xiao, "Geography of spin surfaces", Topology 35:4 (1996), 845-862. MR 98h:14046 Zbl 0874.14031

[Reid 1978] M. Reid, "Surfaces with $p_{g}=0, K^{2}=1$ ", J. Fac. Sci. Univ. Tokyo Sect. IA Math. 25:1 (1978), 75-92. MR 80h:14018 Zbl 0399.14025

[Reider 1988] I. Reider, "Vector bundles of rank 2 and linear systems on algebraic surfaces", Ann. of Math. (2) 127:2 (1988), 309-316. MR 89e:14038 Zbl 0663.14010

[Rohlin 1952] V. A. Rohlin, "New results in the theory of four-dimensional manifolds", Doklady Akad. Nauk SSSR (N.S.) 84 (1952), 221-224. In Russian. MR 14,573b

[Smith 2000] I. Smith, "On moduli spaces of symplectic forms", Math. Res. Lett. 7:5-6 (2000), 779-788. MR 2002a:53112 Zbl 1010.53063

[Taubes 1995a] C. H. Taubes, "More constraints on symplectic forms from Seiberg-Witten invariants”, Math. Res. Lett. 2:1 (1995), 9-13. MR 96a:57075 Zbl 0854.57019

[Taubes 1995b] C. H. Taubes, "The Seiberg-Witten and Gromov invariants", Math. Res. Lett. 2:2 (1995), 221-238. MR 96a:57076 Zbl 0854.57020

[Thurston 1976] W. P. Thurston, "Some simple examples of symplectic manifolds", Proc. Amer. Math. Soc. 55:2 (1976), 467-468. MR 53 \#6578 Zbl 0324.53031

[Vidussi 2001] S. Vidussi, "Homotopy K3's with several symplectic structures", Geom. Topol. 5 (2001), 267-285. MR 2002a:57045 Zbl 1067.57031

[Vidussi 2007] S. Vidussi, "On the number of components of the symplectic representatives of the canonical class”, J. Eur. Math. Soc. 9:4 (2007), 789-800. MR 2008g:57030 Zbl 1144.57023

[Witten 1994] E. Witten, "Monopoles and four-manifolds", Math. Res. Lett. 1:6 (1994), 769-796. MR 96d:57035 Zbl 0867.57029

Received January 29, 2009.

MARK J. D. HAMiLTON

MATHEMATISCHES INSTITUT

LUDWIG-MAXIMILIANS-UNIVERSITÄT MÜNCHEN

THERESIENSTRASSE 39

80333 MUNICH

GERMANY

mark.hamilton@math.lmu.de 